\title{
MAXIMUM BOUNDARY REGULARITY OF BOUNDED HUA-HARMONIC FUNCTIONS ON TUBE DOMAINS
}

\author{
ALINE BONAMI, DARIUSZ BURACZEWSKI ${ }^{1}$, EWA DAMEK ${ }^{1}$, ANDRZEJ HULANICKI ${ }^{1}$ \& PHILIPPE JAMING
}

\begin{abstract}
In this paper we prove that bounded Hua-harmonic functions on tube domains that satisfy some boundary regularity condition are necessarily pluriharmonic. In doing so, we show that a similar theorem is true on one-dimensional extensions of the Heisenberg group or equivalently on the Siegel upper half-plane.
\end{abstract}

\section{INTRODUCTION}

Let $\mathcal{U}^{n} \subset \mathbb{C}^{n+1}$ be the Siegel upper half-plane, defined by

$$
\mathcal{U}^{n}=\left\{z \in \mathbb{C}^{n+1}: \operatorname{Im} z_{n+1}>\sum_{j=1}^{n}\left|z_{j}\right|^{2}\right\} .
$$

Let $F$ be the Poisson-Szegö integral of some boundary function $f$. It has been known for a long time now that $F$, which is smooth inside $\mathcal{U}^{n}$, cannot have bounded transversal Euclidean derivatives up to the boundary, unless $F$ is a pluriharmonic function. More precisely, in $\mathcal{U}^{n}$ we introduce coordinates $\zeta=\left(z_{1}, \ldots, z_{n}\right), z_{n+1}=t+i|\zeta|^{2}+i a$, with $t \in \mathbb{R}$, and $a>0$, so that, for the complex structure $J$, we have $J \partial_{a}=-\partial_{t}$. Then, if $F$ is harmonic with respect to the invariant Laplacian, the derivatives $\partial_{a}^{k} F\left(\zeta, t+i|\zeta|^{2}+i a\right)$ cannot be bounded for large $k$ unless $F$ is pluriharmonic. Such results may be found in the work of Graham (see [Gr1] and Gr2]). The conditions are easier to describe for functions on the unit ball in $\mathbb{C}^{n+1}$, that is, for the bounded realization of $\mathcal{U}^{n}$, see [BBG]. In both cases, a central role is played by the invariant Laplacian $L$. Indeed, Poisson-Szegö integrals are characterized by the fact that they are annihilated by $L$ so that these results are merely results about $L$-harmonic functions.

The aim of this paper is to show that this property holds in a general context. More precisely, we consider an irreducible symmetric Siegel domain of tube type which may be written as

$$
\mathcal{D}=V+i \Omega \subset V^{\mathbb{C}},
$$

where $V$ is a real Euclidean space of dimension $m$ and $\Omega$ is an irreducible symmetric cone inside $V$. Typical examples are given when one chooses for $\Omega$ the forward light cone or the cone of positive definite matrices. For such domains, Poisson-Szegö integrals have been characterized in terms of differential operators of order 2. Let us give more details, and introduce the Hua system which plays the same role as the invariant Laplacian in the case of $\mathcal{U}^{n}$. This system can be defined geometrically for a more general domain $\mathcal{D}$ in $\mathbb{C}^{m}$ which is biholomorphically equivalent to a bounded domain. For

1991 Mathematics Subject Classification. 22E30;32M15;35J25;58J32.

Key words and phrases. Hua-harmonic functions; boundary regularity; Tube domains; pluriharmonic functions; Heisenberg group.

Research partially financed by the European Commission IHP Network 2002-2006 Harmonic Analysis and Related Problems (Contract Number: HPRN-CT-2001-00273 - HARP).

${ }^{1}$ The authors were partly supported by KBN grant 5 PO3A 02821 and Foundation for Polish Sciences Subsidy $3 / 99$. 
more details we refer to BBDHPT] where the following construction, which is inspired by the work of Wallach, is thoroughly discussed.

Let $T^{\mathbb{C}}$ be the complexified tangent bundle of the complex domain $\mathcal{D}$, let $\mathcal{J}$ be the complex structure, and let $T^{1,0}$ and $T^{0,1}$ be the eigenspaces of $\mathcal{J}$ such that $\left.\mathcal{J}\right|_{T^{1,0}}=i \operatorname{Id},\left.\mathcal{J}\right|_{T^{0,1}}=-i$ Id. The Riemannian connection $\nabla$ induced by the Bergman metric on $\mathcal{D}$ preserves $T^{1,0}(\mathcal{D})$ and so does the curvature tensor $R(Z, W)$. For $Z, W$ two complex vector fields we denote by $R(Z, W)=\nabla_{Z} \nabla_{W}-$ $\nabla_{W} \nabla_{Z}-\nabla_{[Z, W]}$ the curvature tensor restricted to $T^{1,0}(\mathcal{D})$. For $f$ a smooth function on $\mathcal{D}$, let

$$
\Delta(Z, W) f=\left(Z \bar{W}-\nabla_{Z} \bar{W}\right) f=\left(\bar{W} Z-\nabla_{\bar{W}} Z\right) f .
$$

Then $\Delta(Z, W)$ annihilates both holomorphic and anti-holomorphic functions, and consequently, the pluriharmonic functions.

Given an orthonormal frame $E_{1}, E_{2}, \cdots, E_{m}$ of $T^{1,0}(\mathcal{D})$ for the canonical Hermitian product associated to the Bergman metric, the Hua system is defined as follows:

$$
\mathbb{H} f=\sum_{j, k}\left(\Delta\left(E_{j}, E_{k}\right) f\right) R\left(\bar{E}_{j}, E_{k}\right) .
$$

The Hua system does not depend on the choice of the orthonormal frame and it is invariant with respect to biholomorphisms. By definition, $\mathbb{H}$-harmonic functions are functions which are annihilated by $\mathbb{H}$.

When $\mathcal{D}$ is a symmetric Siegel domain of tube type, this system is known to characterize the Poisson-Szegö integrals (see [FK] and [JK]). This means that a function on $\mathcal{D}$ is $\mathbb{H}$-harmonic if, and only if, it is the Poisson-Szegö integral of a hyperfunction on the Shilov boundary.

Our main theorem, may then be stated as follows:

Main Theorem. Let $\mathcal{D}$ be an irreducible symmetric domain of tube type. There exists $k$ (depending on the dimension and the rank) such that, if $F$ is a bounded $\mathbb{H}$-harmonic and has bounded derivatives up to the order $k$, then $F$ is pluriharmonic.

More precise and weaker conditions are given in the sequel of the paper. They are linked to the description of the domain in terms of a solvable group of linear automorphisms and may be expressed in the distribution sense. Roughly speaking, we show that there exists an open dense subset $\widetilde{\partial \mathcal{D}}$ of $\partial \mathcal{D}$, such that for every point $p \in \widetilde{\partial \mathcal{D}}$ there is an open neighborhood of $p$ in $V+i V$, called $U$, and a smooth foliation $U_{a}, a \in[0, \epsilon]$, of $U \cap \overline{\mathcal{D}}$ such that $U_{0}=\partial \mathcal{D} \cap U$ and $J \partial_{a}$ is tangential to $U_{a}$. Moreover, $\widetilde{\partial \mathcal{D}}$ is invariant under a group of biholomorphisms of $\mathcal{D}$ acting transitively on the family of foliations. Locally, the parameter $a$ plays the same role as the coordinate $a$ in the Siegel upper half-plane. If $F$ is Hua-harmonic and for large $k, \partial_{a}^{k} F(\cdot, a)$ is bounded as $a \rightarrow 0$ for all such parameters $a$, then we prove that $F$ is pluriharmonic.

Let us remark that many sufficient conditions for pluriharmonicity, which can be written in terms of families of second order operators, have been given by some of the authors (see [BDH] and [DHMP]). We rely deeply on this previous work. Another main tool of the proof is the fact that we can reduce to the same kind of problem on the domain $\mathcal{U}^{n}$ for a variant of the invariant Laplacian $L$. So, we are lead to consider a whole family of second order operators on $\mathcal{U}^{n}$, and we give sufficient conditions so that functions which are annihilated by them are pluriharmonic. A key tool for this is the existence of a boundary equation which implies pluriharmonicity.

One may ask whether this type of results can be obtained in the more general setting of irreducible symmetric Siegel domains, which contains both those of tube type as well as the Siegel upper half-plane $\mathcal{U}^{n}$, which is of type II. Unfortunately, much less is known for higher rank and type II. In particular, for the Hua system that we described, $\mathbb{H}$-harmonic functions are already pluriharmonic (see [BBDHPT] and B]), and such tools are missing for studying Poisson-Szegö integrals (see BBDHPT for more comments). So, the kind of proof that we are giving here cannot be adapted to such a general situation. 
Let us mention that the same phenomenon has also been studied by B. Trojan, using a direct computation of the Poisson-Szegö kernel and looking at its singularities (see [Tr]).

The article is organized as follows. In the next section, we introduce all necessary notations on the Heisenberg group and its one-dimensional extensions. We then prove a characterization of bounded pluriharmonic functions in terms of boundary conditions and give sufficient regularity conditions for our harmonic functions which imply pluriharmonicity. In the next section, we give all preliminaries that we need on Siegel domains of tube type, including a precise expression of Hua operators in terms of the description of the cone using Jordan algebras. Finally we prove that Hua-harmonic operators have a maximum boundary regularity, unless they are pluriharmonic.

Some technical results on special functions, as well as another proof of the main theorem, are given in the appendix.

\section{The theorem on the Heisenberg Group}

2.1. Preliminaries on the Heisenberg group. In this section we recall some notations and results on the Heisenberg group. Our basic reference is [St]. We will try to keep self-contained up to some results in the two last chapters of that book.

The Heisenberg group $\mathbf{H}^{n}$ is the set

$$
\mathbf{H}^{n}=\mathbb{C}^{n} \times \mathbb{R}=\{[\zeta, t]: \zeta \in \mathbb{C}, t \in \mathbb{R}\}
$$

endowed with the multiplication law

$$
[\zeta, t] \cdot[\eta, s]=[\zeta+\eta, t+s+2 \operatorname{Im}(\zeta \bar{\eta})] .
$$

We write $[\zeta, t]$ for a typical element of $\mathbf{H}^{n}$ and $\zeta=\left(\zeta_{1}, \ldots, \zeta_{n}\right)=\left(x_{1}+i y_{1}, \ldots, x_{n}+i y_{n}\right) \in \mathbb{C}^{n}$.

The Euclidean Lebesgue measure $d x d y d t$, which we will note as well $d \zeta d t$ or $d \omega$ if $\omega=[\zeta, t]$, is both left and right invariant under the action of $\mathbf{H}^{n}$. Convolution on $\mathbf{H}^{n}$ is then given by

$$
f * g([\zeta, t])=\int_{\mathbf{H}^{n}} f([\eta, s]) g\left([\eta, s]^{-1}[\zeta, t]\right) d \eta d s .
$$

We next consider the domain $\mathcal{U}^{n} \subset \mathbb{C}^{n+1}$ and its boundary $b \mathcal{U}^{n}$ defined by

$$
\mathcal{U}^{n}=\left\{z \in \mathbb{C}^{n+1}: \operatorname{Im} z_{n+1}>\sum_{j=1}^{n}\left|z_{j}\right|^{2}\right\}, \quad b \mathcal{U}^{n}=\left\{z \in \mathbb{C}^{n+1}: \operatorname{Im} z_{n+1}=\sum_{j=1}^{n}\left|z_{j}\right|^{2}\right\} .
$$

A typical element of $\mathcal{U}^{n}$ or $b \mathcal{U}^{n}$ is denoted by $z=\left(z^{\prime}, z_{n+1}\right)$. The Heisenberg group $\mathbf{H}^{n}$ acts on $\mathcal{U}^{n}$ and $b \mathcal{U}^{n}$ by

$$
[\zeta, t]\left(z^{\prime}, z_{n+1}\right)=\left(z^{\prime}+\zeta, z_{n+1}+t+2 i z^{\prime} \bar{\zeta}+i|\zeta|^{2}\right) .
$$

Moreover, this action is simply transitive on the boundary $b \mathcal{U}^{n}$ which allows us to identify elements of $\mathbf{H}^{n}$ with elements of $b \mathcal{U}^{n}$ by the action of $\mathbf{H}^{n}$ on the origin $(0,0)$ :

$$
\mathbf{H}^{n} \ni[\zeta, t] \mapsto[\zeta, t](0,0)=\left(\zeta, t+i|\zeta|^{2}\right) \in b \mathcal{U}^{n} .
$$

Further, we write $r(z)=\operatorname{Im}\left(z_{n+1}\right)-\left|z^{\prime}\right|^{2}$, so that $\mathcal{U}^{n}=\{z: r(z)>0\}$ and $b \mathcal{U}^{n}=\{z: r(z)=0\}$. The Heisenberg group $\mathbf{H}^{n}$ acts simply transitively on each level set so that each one of them can be identified with $\mathbf{H}^{n}$.

Next, we consider on $\mathbf{H}^{n}$ the left-invariant vector fields

$$
X_{j}=\frac{\partial}{\partial x_{j}}+2 y_{j} \frac{\partial}{\partial t}, \quad Y_{j}=\frac{\partial}{\partial y_{j}}-2 x_{j} \frac{\partial}{\partial t}, \quad T=\frac{\partial}{\partial t} .
$$

One has $\left[Y_{j}, X_{k}\right]=4 \delta_{j, k} T$ while all other commutators vanish. 
We define the holomorphic and anti-holomorphic left invariant vector fields

$$
\bar{Z}_{j}=\frac{1}{2}\left(X_{j}+i Y_{j}\right)=\frac{\partial}{\partial \bar{\zeta}_{j}}-i \zeta_{j} \frac{\partial}{\partial t}, \quad Z_{j}=\frac{1}{2}\left(X_{j}-i Y_{j}\right)=\frac{\partial}{\partial \bar{\zeta}_{j}}+i \bar{\zeta}_{j} \frac{\partial}{\partial t}
$$

so that $\left[\bar{Z}_{j}, Z_{k}\right]=2 i \delta_{j, k} T$ while all other commutators are 0 .

The following operators then play a fundamental role in complex analysis on $\mathcal{U}^{n}$ (see $\mathrm{St}$ ):

$$
\mathcal{L}_{\alpha}=-\frac{1}{2} \sum_{j=1}^{n}\left(Z_{j} \bar{Z}_{j}+\bar{Z}_{j} Z_{j}\right)+i \alpha T=-\frac{1}{4} \sum_{j=1}^{n}\left(X_{j}^{2}+Y_{j}^{2}\right)+i \alpha T,
$$

where $\alpha \in \mathbb{R}$ is a parameter. We will simply write $\mathcal{L}$ for $\mathcal{L}_{0}$.

The Cauchy kernel on $\mathbf{H}^{n}$ is then given, for $[\zeta, t] \neq[0,0]$, by $K([\zeta, t])=c\left(t+i|\zeta|^{2}\right)^{-n-1}$ with $c=2^{n-1} i^{n+1} n ! / \pi^{n+1}$ and the Cauchy-Szegö projection is defined by $C(f)=f * K$, where $K$ defines as well the principal value distribution which is associated to this kernel.

Define

$$
\Phi([\zeta, t])=\frac{2^{n-2}(n-1) !}{\pi^{n+1}} \log \left(\frac{|\zeta|^{2}-i t}{|\zeta|^{2}+i t}\right)\left(|\zeta|^{2}-i t\right)^{-n}
$$

where

$$
\log \left(\frac{|\zeta|^{2}-i t}{|\zeta|^{2}+i t}\right)=\log \left(|\zeta|^{2}-i t\right)-\log \left(|\zeta|^{2}+i t\right)
$$

and the logarithms are taken to be their principal branch in the right half-plane. Write $\widetilde{S}(f)=f * \Phi$. From Formula (53) page 616 in $[\mathrm{St}]$, we then know that if $f$ is a smooth compactly supported function, then

$$
\mathcal{L}_{n} \widetilde{S}(f)=\widetilde{S}\left(\mathcal{L}_{n} f\right)=f-C(f) .
$$

2.2. One dimensional extension of the Heisenberg group. We now define the non-isotropic dilations on $\mathcal{U}^{n}$ and $b \mathcal{U}^{n}$ : for $\delta>0$ and $z=\left(z^{\prime}, z_{n+1}\right) \in \mathcal{U}^{n}$ or $b \mathcal{U}^{n}$, we write

$$
\delta \circ z=\left(\delta z^{\prime}, \delta^{2} z_{n+1}\right) \text {. }
$$

These dilations preserve $\mathcal{U}^{n}$ and $b \mathcal{U}^{n}$ whereas $r(\delta \circ z)=\delta^{2} r(z)$.

We now consider the semi-direct extension of $\mathbf{H}^{n}, S=\mathbf{H}^{n} \mathbb{R}_{*}^{+}$where the action of $\mathbb{R}_{*}^{+}$on $\mathbf{H}^{n}$ is given by $a[\zeta, t]=\left[a^{1 / 2} \zeta, a t\right]$. We will write $[\zeta, t, a]=[[\zeta, t], a]$ for a typical element of $S$ with $[\zeta, t] \in \mathbf{H}^{n}$ and $a \in \mathbb{R}_{*}^{+}$. The group law of $S$ is then given by

$$
[\zeta, t, a][\eta, s, b]=\left[[\zeta, t]\left[a^{1 / 2} \eta, a s\right], a b\right]=\left[\zeta+a^{1 / 2} \eta, t+a s+2 a^{1 / 2} \operatorname{Im}(\bar{\zeta} \eta), a b\right] .
$$

We then extend the definition of the previous vector fields from $\mathbf{H}^{n}$ to $S$ so that the following are left-invariant vector fields on $S$ :

$$
a^{1 / 2} X_{j}, a^{1 / 2} Y_{j}, a T, a \partial_{a}=a \frac{\partial}{\partial a}, a^{1 / 2} Z_{j}, a^{1 / 2} \bar{Z}_{j}
$$

We next define $Z_{n+1}=\frac{1}{2}\left(T-i \partial_{a}\right)$ and $\bar{Z}_{n+1}=\frac{1}{2}\left(T+i \partial_{a}\right)$ so that $a Z_{n+1}$ and $a \bar{Z}_{n+1}$ are left-invariant vector fields on $\mathcal{U}_{n}$ (this notation differs from that in [St] by a harmless factor $2^{1 / 2} i$ ).

We get $\left[a^{1 / 2} X_{j}, a^{1 / 2} Y_{j}\right]=4 \delta_{j, k} a T,\left[a \partial_{a}, a^{1 / 2} X_{j}\right]=\frac{1}{2} a^{1 / 2} X_{j},\left[a \partial_{a}, a^{1 / 2} Y_{j}\right]=\frac{1}{2} a^{1 / 2} Y_{j}\left[a \partial_{a}, a T\right]=$ $a T$, while all other commutators are 0 .

The group $S$ acts simply transitively on $\mathcal{U}^{n}$ by $[0,0, a] z=a^{1 / 2} \circ z$ and $[\zeta, t, 1] z=[\zeta, t] z$ as defined previously. In other words

$$
[\zeta, t, a] z=[\zeta, t, 1][0,0, a] z=[\zeta, t]\left(a^{1 / 2} z^{\prime}, a z_{n+1}\right)=\left(\zeta+a^{1 / 2} z^{\prime}, t+a z_{n+1}+2 i a^{1 / 2} z^{\prime} \bar{\zeta}+i|\zeta|^{2}\right) .
$$


In particular $[\zeta, t, a](0, i)=\left(\zeta, t+i|\zeta|^{2}+i a\right)$. This allows us to identify $S$ with $\mathcal{U}^{n}$ and a function $f_{s}$ on $S$ with a function $f_{u}$ on $\mathcal{U}^{n}$ by

$$
f_{s}([\zeta, t, a])=f_{u}\left(\zeta, t+i|\zeta|^{2}+i a\right) \quad \text { and } \quad f_{u}\left(z^{\prime}, z_{n+1}\right)=f_{s}\left(\left[z^{\prime}, \operatorname{Re}\left(z_{n+1}\right), \operatorname{Im}\left(z_{n+1}\right)-\left|z^{\prime}\right|^{2}\right]\right) .
$$

It then follows that, for $k=1, \ldots, n, \frac{\partial f_{u}}{\partial z_{k}}=Z_{k} f_{s}-2 i \bar{z}_{k} Z_{n+1} f_{s}$. We will thus say that

- $f_{s}$ is holomorphic if $f_{u}$ is holomorphic, i.e. $\frac{\partial f_{u}}{\partial \bar{z}_{k}}=0$ for $k=1, \ldots, n+1$. This is easily seen to be equivalent to $\overline{Z_{k}} f_{s}=0$ for $k=1, \ldots, n+1$.

- $f_{s}$ is anti-holomorphic if $f_{u}$ is anti-holomorphic, i.e. $\frac{\partial f_{u}}{\partial z_{k}}=0$ for $k=1, \ldots, n+1$ or equivalently if $Z_{k} f_{s}=0$ for $k=1, \ldots, n+1$.

- $f_{s}$ is pluriharmonic if $f_{u}$ is pluriharmonic, i.e. $\frac{\partial}{\partial z_{k}} \frac{\partial}{\partial \bar{z}_{j}} f_{u}=0$ for $j, k=1, \ldots, n+1$. This is then equivalent to $Z_{k} \bar{Z}_{j} f_{s}=0$ for $1 \leq j \neq k \leq n+1, Z_{n+1} \bar{Z}_{n+1} f_{s}=0$ and $\left(Z_{k} \bar{Z}_{k}+2 i \bar{Z}_{n+1}\right) f_{s}=0$ for $k=1, \ldots, n$.

In the sequel, we will drop the subscripts $s$ and $u$ for simplicity as well as the superfluous brackets [,]. Notation. Let us fix $\alpha>0$ and consider the left-invariant operator $L_{\alpha}$ given by

$$
L_{\alpha}=-\alpha a\left(\mathcal{L}+n \partial_{a}\right)+a^{2}\left(\partial_{a}^{2}+T^{2}\right) .
$$

Of particular interest in the next section will be the case $\alpha=\frac{1}{2}$.

Let $P_{a}^{\alpha}$ be the Poisson kernel for $L_{\alpha}$, i.e. the function on $\mathbf{H}^{n}$ that establishes a one-to-one correspondence between bounded functions $f$ on $\mathbf{H}^{n}$ and bounded $L_{\alpha}$-harmonic functions $F$ on $S$ by

$$
F(\omega, a)=\int_{\mathbf{H}^{n}} f(\mathrm{w}) P_{a}^{\alpha}\left(\mathrm{w}^{-1} \omega\right) d \mathrm{w}=f * P_{a}^{\alpha}(\omega) .
$$

We are now in position to prove the following theorem, which gives characterizations of boundary values of holomorphic or pluriharmonic $L_{\alpha}$-harmonic functions in terms of differential equations. Let us mention that the existence of such differential equations is well known with different assumptions on the function (see for instance [L1] or Gr1, Gr2]), and the fact that it may be generalized to bounded functions is implicit in the work of different authors, at least when $\alpha=1$, which corresponds to the invariant Laplacian. Our aim, here, is to give a complete proof for bounded functions. It may simplify previous proofs where such boundary differential equation appeared.

Theorem 2.1. Let $F$ be a bounded $L_{\alpha}$-harmonic function on $\mathcal{U}_{n}$ with boundary value $f$.

(i) Then $F$ is holomorphic if and only if $\mathcal{L}_{n} f=0$.

(ii) Then $F$ is anti-holomorphic if and only if $\mathcal{L}_{-n} f=0$.

(iii) Then $F$ is pluriharmonic if and only if $\mathcal{L}_{-n} \mathcal{L}_{n} f=\left(\mathcal{L}^{2}+n^{2} T^{2}\right) f=0$.

Proof. In each case, one implication is elementary. We will content ourselves to prove the converse, that is, solutions of the boundary equations are holomorphic (resp. anti-holomorphic, resp. pluriharmonic).

The key point is the following lemma.

Lemma 2.2. Let $f$ be a smooth bounded function on $\mathbf{H}^{n}$ such that $\mathcal{L}_{n} f=0$. Then $f$ satisfies the boundary Cauchy-Riemann equations, that is,

$$
\bar{Z}_{k} f=0 \text { for } k=1, \ldots n \text {. }
$$

Proof. Replacing eventually $f$ by a left translate of $f$, it is sufficient to prove it at the point $[0,0]$.

Let us now consider $\varphi$ a smooth compactly supported function on $\mathbf{H}^{n}$ such that $\varphi=1$ in a neighborhood of $[0,0]$. Let $\varphi_{R}(\zeta, t)=\varphi\left(R^{-1} \zeta, R^{-2} t\right)$. We may apply Formula (2.1) to $\varphi_{R} f$ to get $\varphi_{R} f=C\left(\varphi_{R} f\right)+\widetilde{S}\left(\mathcal{L}_{n} \varphi_{R} f\right)$. The first term satisfies the boundary Cauchy-Riemann equations, and 
we are reduced to consider the second term. As $\mathcal{L}_{n}\left(\varphi_{R} f\right)=0$ in some anisotropic ball of radius $c R$, it is equal to some $\psi_{R} \mathcal{L}_{n}\left(\varphi_{R} f\right)$ where $\psi_{R}(\zeta, t)=\psi\left(R^{-1} \zeta, R^{-2} t\right)$ and $\psi=0$ in the ball of radius $c$. Finally,

$$
\begin{aligned}
\bar{Z}_{k} f(0,0) & =\bar{Z}_{j} \varphi_{R} f(0,0)=\bar{Z}_{k} \widetilde{S}\left(\psi_{R} \mathcal{L}_{n}\left(\varphi_{R} f\right)\right)(0,0) \\
& =\int_{\mathbf{H}^{n}} \bar{Z}_{k} \Phi(\eta, s) \mathcal{L}_{n}\left(\varphi_{R} f\right)(\eta, s) \psi_{R}(\eta, s) d \eta d s \\
& =\int_{\mathbf{H}^{n}} \mathcal{L}_{-n}\left(\psi_{R} \bar{Z}_{k} \Phi\right)\left(\varphi_{R} f\right) d \eta d t .
\end{aligned}
$$

But $\varphi_{R} f$ is bounded, while an elementary computation gives that $\mathcal{L}_{-n}\left(\psi_{R} \bar{Z}_{k} \Phi\right)$ is bounded by $R^{-3-2 n}$. Since we integrate over a shell of volume $R^{2 n+2}$, letting $R \rightarrow \infty$, we get $\bar{Z}_{k} f(0,0)=0$.

Let us now come back to the proof of the theorem (part (l)). Let us consider a function $F$ such that its boundary value $f$ is annihilated by $\mathcal{L}_{n}$ and show that it is holomorphic. We claim that it is sufficient to find a bounded holomorphic function $G$ that has the same boundary values: indeed, $G$ is also $L_{\alpha}$-harmonic, and so $G=F$. Let us now take for $G$ the unique bounded solution of the equation $\mathcal{L} G+n \partial_{a} G=0$ on $\mathcal{U}_{n}$, with $G$ given by $f$ on the boundary. Commutation properties of $\mathcal{L}, T$ and $\partial_{a}$ give that $\mathcal{L}_{n} G=0$. It follows directly, using the two equations satisfied by $G$, that $\partial_{a} G-i T G=0$, that is, $\bar{Z}_{n+1} G=0$. Moreover, since on each level set $\left\{r(z)=a_{0}\right\}$ the function $G$ is smooth, bounded, and satisfies the equation $\mathcal{L}_{n} G=0$, we also know from the lemma that $\bar{Z}_{k} G=0$ for $k=1,2, \cdots, n$. We have proved that $G$, and so $F$, is holomorphic.

Part (ii) is obtained by conjugation.

Let us now turn to assertion (iii) of Theorem 2.1. We know from part (iii) that $\mathcal{L}_{n} f$ is antiholomorphic. First, note that $\mathcal{L}_{n} Z_{n+1} F=Z_{n+1} \mathcal{L}_{n} F=0$. It follows from part (ii) that

$$
\bar{Z}_{j} Z_{n+1} F=0 \text { for } j=1, \ldots, n+1 \text {. }
$$

Further, for $k=1, \ldots, n$, note that

$$
\begin{aligned}
{\left[\frac{\partial}{\partial z_{k}}, Z_{k} \bar{Z}_{k}\right] F } & =\left[\left(Z_{k}-2 i \bar{z}_{k} Z_{n+1}\right), Z_{k} \bar{Z}_{k}\right] F \\
& =Z_{k}\left[Z_{k}, \bar{Z}_{k}\right] F+2 i\left(Z_{k} \bar{z}_{k}\right)\left(\bar{Z}_{k} Z_{n+1} F\right)+Z_{k}\left(\left(\bar{Z}_{k} \bar{z}_{k}\right) Z_{n+1}\right) F .
\end{aligned}
$$

But $\left[Z_{k}, \bar{Z}_{k}\right]=-2 i T, \bar{Z}_{k} Z_{n+1} f=0$ and $\bar{Z}_{k} \bar{z}_{k}=1$ so that

$$
\left[\frac{\partial}{\partial z_{k}}, Z_{k} \bar{Z}_{k}\right] F=-2 i Z_{k} T F+2 i Z_{k} Z_{n+1} F=-2 i Z_{k} T f+2 i Z_{k}\left(Z_{n+1}+\bar{Z}_{n+1}\right) F=0 .
$$

It follows that $\mathcal{L}_{n} \frac{\partial}{\partial z_{k}} F=\frac{\partial}{\partial z_{k}} \mathcal{L}_{n} F=0$ which by part (ii) implies that $\frac{\partial}{\partial z_{k}} F$ is holomorphic so that $F$ is pluriharmonic.

Remark. This theorem is false if one removes the boundedness property of $F$. For example, $F[\zeta, t, a]=$ $a^{n \alpha+1}$ is $L_{\alpha}$-harmonic on $\mathbb{H} \times \mathbb{R}$. Its boundary value $f[\zeta, t]=0$ satisfies $\left(\mathcal{L}^{2}+T^{2}\right) f=0$ on the boundary but is not pluriharmonic.

2.3. Pluriharmonicity of centrally-independent bounded boundary-regular functions. In a first step, we will consider $L_{\alpha}$-harmonic functions $f$ that do not depend on the central variable $t$, that is, functions which satisfy $L_{\alpha} f=0$ and $T f=0$. Since $t$ acts as a parameter, it follows directly from the analysis of the corresponding situation on $\mathbb{C}^{n}$. We first compute explicitely the corresponding Poisson kernel.

Notation. Let $\Delta$ be the standard Laplacian on $\mathbb{C}^{n}$ (identified with $\mathbb{R}^{2 n}$ ). For $\alpha>0$, let

$$
\Lambda_{\alpha}=\alpha a\left(\Delta-n \partial_{a}\right)+a^{2} \partial_{a}^{2}
$$


be an operator on $\mathbb{C}^{n}$. Let $Q_{a}^{\alpha}$ be the Poisson kernel for $\Lambda_{\alpha}$. A bounded $\Lambda_{\alpha}$-harmonic function $F$ over $\mathbb{C}^{n}$ is given by

$$
F(\zeta)=f *_{\mathbb{C}^{n}} Q_{a}^{\alpha}(\zeta)=\int_{\mathbb{C}^{n}} f\left(\zeta^{\prime}\right) Q_{a}^{\alpha}\left(\zeta-\zeta^{\prime}\right) d \zeta^{\prime}
$$

with $f \in L^{\infty}\left(\mathbb{C}^{n}\right)$.

Let us first identify the Fourier transform over $\mathbb{C}^{n}$ of $Q_{a}^{\alpha}$.

Lemma 2.3. The Fourier transform $\widehat{Q_{a}^{\alpha}}$ of the kernel $Q_{a}^{\alpha}$ is given by $\widehat{Q_{a}^{\alpha}}(\xi)=z\left(\alpha|\xi|^{2} a\right)$, where $z$ is the unique bounded solution of the equation

$$
\left(-1-\alpha n \partial_{a}+a \partial_{a}^{2}\right) z(a)=0
$$

with $z(0)=1$. In particular, $\widehat{Q_{a}^{\alpha}}$ is a smooth function on $\mathbb{C}^{n} \backslash\{0\}$.

Proof. The properties of solutions of Equation (2.3) are given in Appendix B. In particular, this equation has a unique bounded solution with $z(0)=1$.

Next, observe that $a \rightarrow \widehat{Q_{a}}(\xi)$ is a bounded solution of the equation

$$
\left(-\alpha|\xi|^{2}-\alpha n \partial_{a}+a \partial_{a}^{2}\right) y(a)=0 .
$$

With the change of variables $y(a)=z\left(\alpha|\xi|^{2} a\right), \xi$ fixed, this equation transforms into (2.3) so that there exists $c(\xi)$ such that $\widehat{\widetilde{P}}_{a}(\xi)=c(\xi) z\left(\alpha|\xi|^{2} a\right)$.

Further

$$
c(\xi)=\lim _{a \rightarrow 0} \widehat{Q_{a}}(\xi)=1,
$$

which allows to conclude.

Let us now state the main result of this section.

Proposition 2.4. Let $\alpha>0$ and $k$ be the smallest integer bigger than na. Let $F$ be a bounded $\Lambda_{\alpha}$-harmonic function on $\mathbb{C}^{n}$ that satisfies the following boundary regularity condition: for every $p=0, \ldots, k+1$ and every $\varphi \in \mathcal{S}\left(\mathbb{C}^{n}\right)$,

$$
\sup _{a \leq 1}\left|\int_{\mathbb{C}^{n}} \partial_{a}^{p} F(\zeta, a) \varphi(\zeta) d \zeta\right|<\infty
$$

Then $F$ is constant.

Proof. Let us write $F=f *_{\mathbb{C}^{n}} Q_{a}^{\alpha}$, with $f$ a bounded function. Now, let $\varphi \in \mathcal{S}\left(\mathbb{C}^{n}\right)$ be a function such that $\widehat{\varphi}$ has compact support with $0 \notin \operatorname{supp} \widehat{\varphi}$. We claim that it is sufficient to prove that, for all such $\varphi$, we have the identity $\int_{\mathbb{C}^{n}} F_{a}(\zeta) \varphi(\zeta) d \zeta=0$. Indeed, as a consequence, the Fourier transform of $f$ is supported in $\{0\}$. It follows that $f$ is a polynomial. Since $f$ is bounded, then $f$ is constant and finally, $F$ is constant.

By definition of the Fourier transform of $f$ as a distribution, we may write

$$
\int_{\mathbb{C}^{n}} F_{a}(\zeta) \varphi(\zeta) d \zeta=\left\langle\widehat{f}, \widehat{\varphi} \widehat{\widetilde{Q}_{a}^{\alpha}}\right\rangle
$$

whence,

$$
I_{p}(a):=\partial_{a}^{p} \int_{\mathbb{C}^{n}} F_{a}(\zeta) \varphi(\zeta) d \zeta=\partial_{a}^{p}\left\langle\widehat{f}, \widehat{\varphi} \widehat{\widetilde{Q}_{a}^{\alpha}}\right\rangle=\alpha^{p}\left\langle\widehat{f}(\cdot), \widehat{\varphi}(\cdot)|\cdot|^{2 p} z^{(p)}\left(|\cdot|^{2} a\right)\right\rangle
$$


where the function $z$ is given in the previous lemma. From now on, we assume that n $\alpha$ is not an integer. It is then easy to modify the following proof to cover the remaining case. According to Appendix B, for $p=k+1$, we have

$$
\partial_{a}^{p} z\left(\alpha|\xi|^{2} a\right)=\gamma\left(|\xi|^{2} a\right)+a^{n \alpha-k}|\xi|^{2(n \alpha-k)} \widetilde{\gamma}\left(|\xi|^{2} a\right)
$$

with $\gamma, \widetilde{\gamma}$ smooth functions up to 0 of order $N$, and $\widetilde{\gamma}(0) \neq 0$. We choose $N$ large enough, depending on the order of the distribution $\widehat{f}$. Then

$$
I(a) \simeq c a^{n \alpha-k}, \text { with } c=\widetilde{\gamma}(0)\left\langle\widehat{f}(\cdot), \widehat{\varphi}(\cdot)|\cdot|^{2 n \alpha+2}\right\rangle,
$$

unless the constant $c$ vanishes. By assumption, it is a bounded function of $a$. So $c=0$, that is, $\left\langle\widehat{f}(\cdot), \widehat{\varphi}(\cdot)|\cdot|^{2 n \alpha+2}\right\rangle=0$.

Since any function in $\mathcal{S}\left(\mathbb{C}^{n}\right)$ with compact support in $\mathbb{C}^{n} \backslash\{0\}$ can be written as $\widehat{\varphi}(\cdot)|\cdot|^{2 n \alpha+2}$, we conclude that $\widehat{f}$ vanishes outside 0 , which we wanted to prove.

Remark. We may identify functions on $S$ that are independent on $t$ with functions on the hyperbolic upper half-plane $\mathbb{R}^{2 n} \times \mathbb{R}_{*}^{+}$. This one is an unbounded realization of the real hyperbolic space of dimension $2 n+1$, while a bounded realization is given by the real hyperbolic ball $\mathbb{B}_{2 n+1}$ of dimension $2 n+1$. The above theorem is therefore an analogue of Theorem 8 in Ja1 (see also Proposition 2 in Ja2 ), stating that hyperbolic-harmonic functions on $\mathbb{B}_{2 n+1}$ that are regular up to the boundary are constant.

\subsection{Representations of the Heisenberg group and the Poisson kernel for $L_{\alpha}$.}

We now pass to the general case. We will have to use harmonic analysis as before, the scheme of the proof being somewhat similar. Let us first recall some definitions which are linked to harmonic analysis on the Heisenberg group.

For $\lambda \neq 0$, the unitary representation $R^{\lambda}$ of $\mathbf{H}^{n}$ on $L^{2}\left(\mathbb{R}^{n}\right)$ is defined by

$$
R^{\lambda}(\zeta, t) \Phi(x)=e^{2 \pi i \lambda(u . x+u \cdot v / 2+t / 4)} \Phi(x+v)
$$

if we set $\zeta=u+i v$. The Fourier transform of an integrable function $f$ on $\mathbf{H}^{n}$ is then the operatorvalued function $\lambda \mapsto \hat{f}(\lambda)$ given by

$$
\langle\hat{f}(\lambda) \phi, \psi\rangle=\int_{\mathbf{H}^{n}}\left\langle R^{\lambda}(\zeta, t) \phi, \psi\right\rangle f(\zeta, t) d \zeta d t .
$$

Notation. Recall that the Hermite functions $H_{k}$ of one variable are defined by the formula

$$
H_{k}(x)=(-1)^{k} e^{x^{2} / 2}\left(\frac{d}{d x}\right)^{k} e^{-x^{2}}, \quad k=0,1,2, \ldots
$$

For $c_{k}=\left(\sqrt{\pi} 2^{k} k !\right)^{1 / 2}$, we note $h_{k}=c_{k} H_{k}$. This family of functions forms an orthonormal basis of $L^{2}(\mathbb{R})$. For $k=\left(k_{1}, \ldots, k_{n}\right)$ a multi-index, we write $h_{k}=h_{k_{1}} \ldots h_{k_{n}}$.

Now, for $\lambda \neq 0$, denote by $h_{k}^{\lambda}(x)=(2 \pi|\lambda|)^{n / 4} h_{k}\left((2 \pi|\lambda|)^{1 / 2} x\right)$. Finally, let

$$
e_{\kappa}^{\lambda}(\omega)=\sum_{|k|=\kappa}\left(R^{\lambda}(\omega) h_{k}^{\lambda}, h_{k}^{\lambda}\right)
$$

These last functions may be used to write explicitly an inverse Fourier Formula (see [I], or Appendix C). 
Let $P_{a}^{\alpha}$ be the Poisson kernel for $L_{\alpha}$, i.e. the function on $\mathbf{H}^{n}$ that establishes a one-to-one correspondence between bounded functions $f$ on $\mathbf{H}^{n}$ and bounded $L_{\alpha}$-harmonic functions $F$ on $S$ by

$$
F(\omega, a)=\int_{\mathbf{H}^{n}} f(\mathrm{w}) P_{a}^{\alpha}\left(\mathrm{w}^{-1} \omega\right) d \mathrm{w}=f * P_{a}^{\alpha}(\omega),
$$

normalized by $\left\|P_{a}^{\alpha}\right\|_{L^{1}\left(\mathbf{H}^{n}\right)}=1$. Further, for a function $f$ on $\mathbf{H}^{n}$ and $\omega \in \mathbf{H}^{n}$, write ${ }_{\omega} f$ for the function given by ${ }_{\omega} f(\mathrm{w})=f(\omega \mathrm{w})$.

Lemma 2.5. Let $\lambda \neq 0, \kappa$ be an integer, $\alpha>0$ and $\rho=\alpha(2 \kappa+n)$. Fix $\omega \in \mathbf{H}^{n}$ and define

$$
g_{\kappa}^{\lambda}(\omega, a)=\sum_{k:|k|=\kappa}\left\langle\widehat{\omega} \widehat{P}_{a}^{\alpha}(\lambda) h_{k}^{\lambda}, h_{k}^{\lambda}\right\rangle=\int_{\mathbf{H}^{n}} \omega_{a} P_{a}^{\alpha}(\mathrm{w}) e_{\kappa}^{\lambda}(\mathrm{w}) d \mathrm{w} .
$$

Then $g_{\kappa}^{\lambda}(\omega, a)=e_{\kappa}^{\lambda}\left(\omega^{-1}\right) g(|\lambda| a)$, where $g$ is the unique bounded solution on $\mathbb{R}^{+}$of the equation

$$
\left(\partial_{a}^{2}-\frac{\alpha n}{a}-\left(\frac{\rho}{a}+1\right)\right) g(a)=0
$$

with $g(0)=1$.

Proof of Lemma 2.5. As $P_{a}^{\alpha} \in L^{1}\left(\mathbf{H}^{n}\right)$ with $\left\|P_{a}^{\alpha}\right\|_{L^{1}\left(\mathbf{H}^{n}\right)}=1$ and as $e_{\kappa}^{\lambda}$ is easily seen to be bounded, $g_{\kappa}^{\lambda}$ is bounded. Further

$$
\mathcal{L} e_{\kappa}^{\lambda}=(2 \kappa+n)|\lambda| e_{\kappa}^{\lambda} \text {. }
$$

Since $(\mathrm{w}, a) \rightarrow P_{a}^{\alpha}(\omega \mathrm{w})$ is $L_{\alpha}$-harmonic, we have

$$
\int_{\mathbf{H}^{n}} L_{\alpha}\left({ }_{\omega} P_{a}^{\alpha}\right)(\mathrm{w}) e_{\kappa}^{\lambda}(\mathrm{w}) d \mathrm{w}=0 .
$$

By Harnack's inequality, we may interchange the integral with the corresponding differential operators to obtain

$$
\begin{gathered}
\int_{\mathbf{H}^{n}}\left(\partial_{a}^{2}-\frac{\alpha n}{a} \partial_{a}\right){ }_{\omega} P_{a}^{\alpha}(\mathrm{w}) e_{\kappa}^{\lambda}(\mathrm{w}) d \mathrm{w}=\left(\partial_{a}^{2}-\frac{\alpha n}{a} \partial_{a}\right) g_{\kappa}^{\lambda}(\omega, a), \\
\int_{\mathbf{H}^{n}} \mathcal{L}_{\omega} P_{a}(\mathrm{w}) e_{\kappa}^{\lambda}(\mathrm{w}) d \mathrm{w}=\int_{\mathbf{H}^{n}} \omega P_{a}(\mathrm{w}) \mathcal{L} e_{\kappa}^{\lambda}(\mathrm{w}) d \mathrm{w}=(2 \kappa+n)|\lambda| g_{\kappa}^{\lambda}(\omega, a), \\
\int_{\mathbf{H}^{n}} T^{2}{ }_{\omega} P_{a}(\mathrm{w}) e_{\kappa}^{\lambda}(\mathrm{w}) d \mathrm{w}=\int_{\mathbf{H}^{n}}{ }_{\omega} P_{a}(\mathrm{w}) T^{2} e_{\kappa}^{\lambda}(\mathrm{w}) d \mathrm{w}=-\lambda^{2} g_{\kappa}^{\lambda}(\omega, a) .
\end{gathered}
$$

Combining (2.7)-(2.9), we see that the function $g(a)=g_{k}^{\lambda}(\omega, a)$ is a bounded solution of the equation

$$
\left(\partial_{a}^{2}-\frac{\alpha n}{a} \partial_{a}-\left(\frac{\rho|\lambda|}{a}+\lambda^{2}\right)\right) g(a)=0 .
$$

One immediately gets that, for $\omega, \lambda$ fixed, $G_{\omega, \lambda}$ defined by

$$
G_{\omega, \lambda}(a)=g_{\kappa}^{\lambda}\left(\omega^{-1}, a /|\lambda|\right)
$$

satisfies equation (2.6). The fact that this equation has only one bounded solution, up to a constant, is well known (see Appendix A, Lemma A.1). So $g_{\kappa}^{\lambda}\left(\omega^{-1}, a\right)=c_{\kappa}^{\lambda}(\omega) g(|\lambda| a)$ for some function $c_{\kappa}^{\lambda}$.

Moreover, note that $g_{\kappa}^{\lambda}$ may be rewritten

$$
g_{\kappa}^{\lambda}\left(\omega^{-1}, a\right)=e_{k}^{\lambda} * \check{P_{a}^{\alpha}}(\omega),
$$

so that, using the fact that $P_{a}^{\alpha}$ is an approximate identity, by letting $a \rightarrow 0$ in (2.11), we get $c_{\kappa}^{\lambda}=$ $e_{\kappa}^{\lambda}$. 
Remark. Even if we will not use it, let us remark that this allows to write explicitly (see appendix C.2.

$$
P_{a}^{\alpha}(\omega)=c_{n} \int_{\mathbb{R}} \sum_{\kappa \in \mathbb{N}} g_{\kappa}^{\lambda}(\omega, a)|\lambda|^{n} d \lambda
$$

\subsection{An orthogonality property for bounded boundary-regular functions.}

Notation. Given $\psi \in C_{c}^{\infty}(\mathbb{R} \backslash\{0\})$ we define

$$
e_{\kappa}^{\psi}(\mathrm{w})=\int_{\mathbb{R}} e_{\kappa}^{\lambda}(\mathrm{w}) \psi(\lambda) d \lambda
$$

Of course $e_{k}^{\psi}$ is a Schwartz function on $\mathbf{H}^{n}$. We are now in position to prove the following:

Proposition 2.6. Let $f \in L^{\infty}\left(\mathbf{H}^{n}\right)$ and let $F$ be the corresponding bounded $L_{\alpha}$-harmonic function. Let $k$ be the smallest integer bigger then $n \alpha$. Assume that for every $\varphi \in \mathcal{S}\left(\mathbf{H}^{n}\right)$ and every $0 \leq p \leq k+1$,

$$
\sup _{a \leq 1}\left|\int_{\mathbf{H}^{n}} \partial_{a}^{p} F(\mathrm{w}, a) \varphi(\mathrm{w}) d \mathrm{w}\right|<\infty .
$$

Then, for every $\kappa \neq 0$, every $\omega \in \mathbf{H}^{n}$ and every $\psi \in C_{c}^{\infty}(\mathbb{R} \backslash\{0\})$,

$$
\int_{\mathbf{H}^{n}} f(\omega \mathrm{w}) e_{\kappa}^{\psi}(\mathrm{w}) d \mathrm{w}=0
$$

Proof of Proposition 2.0. Property (2.12) is unchanged if one replaces $f$ by $\omega f$ so that it is enough to consider the case $\omega=[0,0]$.

Define

$$
I(a)=\int_{\mathbf{H}^{n}} F(\mathrm{w}, a) e_{\kappa}^{\psi}(\mathrm{w}) d \mathrm{w}=\int_{\mathbf{H}^{n}} f * P_{a}(\mathrm{w}) e_{\kappa}^{\psi}(\mathrm{w}) d \mathrm{w}
$$

where $f$ is the boundary value of $F$. With 2.11) and Lemma 2.5, we get

$$
\begin{aligned}
I(a) & =\int_{\mathbf{H}^{n}} f(\mathrm{w}) \int_{\mathbb{R}} e_{\kappa}^{\lambda} * \check{P}_{a}(\mathrm{w}) \psi(\lambda) d \lambda d \mathrm{w} \\
& =\int_{\mathbf{H}^{n}} f(\mathrm{w}) \int_{\mathbb{R}} e_{\kappa}^{\lambda}(\mathrm{w}) g(|\lambda| a) \psi(\lambda) d \lambda d \mathrm{w} .
\end{aligned}
$$

Using Lemma C.1 in Appendix C, we see that $I$ is a smooth function for $a>0$ and get

$$
\partial^{k+1} I(a)=\int_{\mathbf{H}^{n}} f(\mathrm{w}) \int_{\mathbb{R}} e_{\kappa}^{\lambda}(\mathrm{w})|\lambda|^{k+1} \partial_{a}^{k+1} g(|\lambda| a) \psi(\lambda) d \lambda d \mathrm{w} .
$$

From now on, we assume that $\alpha n$ is not an integer. It is again easy to adapt the proof to the other case. Using Lemma A.1 in Appendix A with $N=1$, can write

$$
\partial^{k+1} g(|\lambda| a)=|\lambda|^{\alpha n-k} a^{\alpha n-k} g_{1}(|\lambda| a)+g_{2}(|\lambda| a)
$$

with $g_{1}$ and $g_{2}$ having continuous derivatives up to 0 , and $g_{1}(0) \neq 0$. Using again Lemma C.1, we can pass to the limit when $a$ tends to 0 in the two integrals. We find that

$$
\partial^{k+1} I(a) \simeq c a^{\alpha n-k} \text { with } c=g_{1}(0) \int_{\mathbf{H}^{n}} f(\mathrm{w}) \int_{\mathbb{R}} e_{\kappa}^{\lambda}(\mathrm{w})|\lambda|^{k+1} \psi(\lambda) d \lambda d \mathrm{w}
$$

unless $c=0$. Now, Hypothesis (2.12) implies that $\partial_{a}^{k+1} I(a)$ is bounded, so it is indeed the case and

$$
\int_{\mathbf{H}^{n}} f(\mathrm{w}) \int_{\mathbb{R}} e_{\kappa}^{\lambda}(\mathrm{w})|\lambda|^{k+1} \psi(\lambda) d \lambda d \mathrm{w}=0 .
$$


Now every function in $\mathcal{C}_{c}^{\infty}(\mathbb{R} \backslash\{0\})$ can be written in the form $|\lambda|^{k+1} \psi(\lambda)$. This completes the proof of the proposition.

2.6. The main theorem on the Heisenberg group. We are now in position to prove our main theorem in this context:

Theorem 2.7. Let $\alpha>0$ and $k$ the smallest integer bigger than n $\alpha$. Let $F$ be a bounded $L_{\alpha}$-harmonic function on $S$. Assume that for every $\varphi \in \mathcal{S}\left(\mathbf{H}^{n}\right)$ and every $0 \leq p \leq k+1$,

$$
\sup _{a \leq 1}\left|\int_{\mathbf{H}^{n}} \partial_{a}^{p} F(\mathrm{w}, a) \varphi(\mathrm{w}) d \mathrm{w}\right|<\infty .
$$

Then, $F$ is pluriharmonic.

Proof. Let $f \in L^{\infty}\left(\mathbf{H}^{n}\right)$ be the boundary value of $F$. We will use the following well-known fact: the pointwise limit of a uniformly bounded sequence of pluriharmonic functions is again pluriharmonic. It allows to replace $f$ by its right convolution with a smooth approximate identity. Moreover, such a function satisfies also (2.12). So, from now on in this proof, we may assume that $f$ is smooth, and that, moreover, its derivatives up to order 4 are bounded.

To prove that $F$ is pluriharmonic, it is sufficient to prove that $g(\mathrm{w})=\left(\mathcal{L}^{2}+n^{2} T^{2}\right) f(\mathrm{w})$ is constant. Indeed, by Harnack's Inequality, such a function tends to 0 when w tends to $\infty$, so $g$ can only be equal to the constant 0 . We then use Theorem 2.1 to conclude.

Let $G$ be the $L_{\alpha}$-harmonic extension of $g$. Then $G$ satisfies also hypothesis (2.12), and according to (2.15) in Proposition 2.6, for every $\kappa \neq 0$, every $\omega \in \mathbf{H}^{n}$ and every $\psi \in \mathcal{C}_{0}^{\infty}(\mathbb{R} \backslash\{0\})$ we have

$$
\int_{\mathbf{H}^{n}} g(\mathrm{w}) e_{\kappa}^{\psi}(\mathrm{w}) d \mathrm{w}=0 .
$$

Moreover, a direct integration by parts, using the fact that

$$
\left(\mathcal{L}^{2}+n^{2} T^{2}\right) e_{0}^{\lambda}=0,
$$

allows to conclude that $(2.15)$ is also valid for $\kappa=0$. We deduce from (C.37) that the Fourier transform of $g$ in the $t$-variable (in the distributional sense) is supported by 0 . It follows that $g$ is a polynomial in the $t$-variable and, as $g$ is bounded, this implies that $g$ is independent of the central variable $t$. According to Proposition 2.4, it follows that $G$ is constant, which we wanted to prove.

2.7. Optimality of the result. We will now prove a converse of Theorem 2.7, showing that the index of regularity given there is optimal. We will actually prove that, in some weak sense, all $L_{\alpha}$-harmonic functions have the regularity just below the limitation given by that theorem.

Recall that a function $F$ on $S$ is said to have distributional boundary value if, for every $\psi \in \mathcal{C}_{c}^{\infty}\left(\mathbf{H}^{n}\right)$, the limit

$$
\lim _{a \rightarrow 0} \int_{\mathbf{H}^{n}} F(\mathrm{w}, a) \psi(\mathrm{w}) d \mathrm{w}
$$

exists. Note that, if $F$ has a boundary distribution, so do $\mathcal{L} F$ and $T F$.

We may now prove the following:

Theorem 2.8. Let $\alpha>0$ and let $k$ be the smallest integer greater then n $\alpha$. Assume $F$ is a $L_{\alpha}$ harmonic function on $S$ with a boundary distribution. Then, for every $p \leq k, \partial_{a}^{p} F$ has a boundary distribution.

Proof. The proof is essentially the same as in Ja2 or BBG which deal with bounded realizations. So we only give a quick outline of it. 
We prove the theorem by induction on $p$. For $p=0$, this is our original assumption. Let us take the statement for granted at rank $p-1$ and fix $\psi \in \mathcal{C}_{c}^{\infty}\left(\mathbf{H}^{n}\right)$. Define

Since

$$
\psi_{p}(a)=\partial_{a}^{p} \int_{\mathbf{H}^{n}} F(\mathrm{w}, a) \psi(\mathrm{w}) d \mathrm{w}=\int_{\mathbf{H}^{n}} \partial_{a}^{p} F(\mathrm{w}, a) \psi(\mathrm{w}) d \mathrm{w} .
$$

applying $\partial_{a}^{p-1}$ to both sides, we get

$$
a \partial_{a}^{2} F-n \alpha \partial_{a} F=\left(\alpha \mathcal{L}+a T^{2}\right) F
$$

$$
a \partial_{a}^{p+1} F+(p-1-n \alpha) \partial_{a}^{p} F=\partial_{a}^{p-1}\left(\alpha \mathcal{L}+a T^{2}\right) F
$$

We know that $\left(\alpha \mathcal{L}+a T^{2}\right) F$ has a boundary distribution. It follows from the induction hypothesis that the second member of (2.17) has a boundary distribution. Now, multiply (2.17) by $\psi$ and integrate over $\mathbf{H}^{n}$, we get that

$$
g_{p}(a):=a \partial \psi_{p}(a)+(p-1-n \alpha) \psi_{p}(a)
$$

has a limit as $a \rightarrow 0$. As $p-1-n \alpha \neq 0$, solving this differential equation, we get

$$
\psi_{p}(a)=\lambda a^{n \alpha-p+1}+a^{n \alpha-p+1} \int_{1}^{a} \frac{g_{p}(t)}{t^{(n \alpha-p+1)+1}} d t .
$$

As $g_{p}$ has a limit when $a \rightarrow 0$, it follows that $\psi_{p}$ has a limit as $a \rightarrow 0$, provided $n \alpha-p+1>0$. We have proved the theorem.

\section{The main theorem on IRReducible Symmetric Siegel Domains of tube type}

We first write the Hua operator in an appropriate coordinate system.

3.1. Preliminaries on irreducible symmetric cones. Let $\Omega$ be an irreducible symmetric cone in an Euclidean space $V$, as in the introduction. We describe precisely the solvable group that acts simply transitively on $\Omega$ in terms of Jordan algebras. We refer to the book of Faraut and Korányi [FK] for these prerequisites, or to [BBDHPT] where a rapid introduction has already been given, with the same notations.

We assume that $V$, endowed with the scalar product $\langle\cdot, \cdot\rangle$ is an Euclidean Jordan algebra, that is, is also endowed with a product such that, for all elements $x, y$ and $z$ in $V$

$$
x y=y x, \quad x\left(x^{2} y\right)=x^{2}(x y), \quad\langle x y, z\rangle=\langle y, x z\rangle .
$$

Moreover, we assume that $V$ is a simple Jordan algebra with unit element $e$. We denote by $L(x)$ the self-adjoint endomorphism of $V$ given by the multiplication by $x$, i.e.

$$
L(x) y=x y .
$$

The irreducible symmetric cone $\Omega$ is then given by

$$
\Omega=\operatorname{int}\left\{x^{2}: x \in V\right\} \text {. }
$$

Let $G$ be the connected component of the group of all transformations in $G L(V)$ which leave $\Omega$ invariant, and let $\mathcal{G}$ be its Lie algebra. Then $\mathcal{G}$ is a subspace of the space of endomorphisms of $V$ which contains all $L(x)$ for all $x \in V$, as well as all $x \square y$ for $x, y \in V$, where

$$
x \square y=L(x y)+[L(x), L(y)]
$$

(see FK for these properties).

We fix a Jordan frame $\left\{c_{1}, \ldots, c_{r}\right\}$ in $V$, that is, a complete system of orthogonal primitive idempotents:

$$
c_{i}^{2}=c_{i}, \quad c_{i} c_{j}=0 \quad \text { if } i \neq j, \quad c_{1}+\ldots+c_{r}=e
$$


and none of the $c_{1}, \ldots, c_{r}$ is a sum of two nonzero idempotents. Let us recall that the length $r$ is the rank of the cone and is independent of the choice of the Jordan frame.

The Peirce decomposition of $V$ related to the Jordan frame $\left\{c_{1}, \ldots, c_{r}\right\}$ ([FK], Theorem IV.2.1) may be written as

$$
V=\bigoplus_{1 \leq i \leq j \leq r} V_{i j}
$$

It is given by the common diagonalization of the self-adjoint endomorphims $L\left(c_{j}\right)$ with respect to their only eigenvalues $0, \frac{1}{2}, 1$. In particular $V_{j j}=\mathbb{R} c_{j}$ is the eigenspace of $L\left(c_{j}\right)$ related to 1 , and, for $i<j, V_{i j}$ is the intersection of the eigenspaces of $L\left(c_{i}\right)$ and $L\left(c_{j}\right)$ related to $\frac{1}{2}$. All $V_{i j}$, for $i<j$, have the same dimension $d$.

For each $i<j$, we fix once for all an orthonormal basis of $V_{i j}$, which we note $\left\{e_{i j}^{\alpha}\right\}$, with $1 \leq \alpha \leq d$. To simplify the notation, we write $e_{i i}^{\alpha}=c_{i}$ ( $\alpha$ taking only the value 1$)$. Then the system $\left\{e_{i j}^{\alpha}\right\}$, for $i \leq j$ and $1 \leq \alpha \leq \operatorname{dim} V_{i j}$, is an orthonormal basis of $V$.

Let us denote by $\mathcal{A}$ the Abelian subalgebra of $\mathcal{G}$ consisting of elements $H=L(a)$, where

$$
a=\sum_{j=1}^{r} a_{j} c_{j} \in \bigoplus_{i} V_{i i}
$$

We set $\lambda_{j}$ the linear form on $\mathcal{A}$ given by $\lambda_{j}(H)=a_{j}$. The Peirce decomposition gives also a simultaneous diagonalization of all $H \in \mathcal{A}$, namely

$$
H x=L(a) x=\frac{\lambda_{i}(H)+\lambda_{j}(H)}{2} x \quad x \in V_{i j} .
$$

Let $A=\exp \mathcal{A}$. Then $A$ is an Abelian group, which is the Abelian part of the Iwasawa decomposition of $G$. We now describe the nilpotent part $N_{0}$. Its Lie algebra $\mathcal{N}_{0}$ is the space of elements $X \in \mathcal{G}$ such that, for all $i \leq j$,

$$
X V_{i j} \subset \bigoplus_{k \geq l ;(k, l)>(i, j)} V_{k l},
$$

where the pairs are ordered lexicographically. Once $\mathcal{N}_{0}$ is defined, we define $\mathcal{S}_{0}$ as the direct sum $\mathcal{N}_{0} \oplus \mathcal{A}$. The groups $S_{0}$ and $N_{0}$ are then obtained by taking the exponentials. It follows from the definition of $\mathcal{N}_{0}$ that the matrices of elements of $\mathcal{S}_{0}$ and $S_{0}$, in the orthonormal basis $\left\{e_{i j}^{\alpha}\right\}$, are upper-triangular.

The solvable group $S_{0}$ acts simply transitively on $\Omega$. This may be found in [FK Chapter VI, as well as the precise description of $\mathcal{N}_{0}$ which will be needed later. One has

$$
\mathcal{N}_{0}=\bigoplus_{i<j \leq r} \mathcal{N}_{i j}
$$

where

$$
\mathcal{N}_{i j}=\left\{z \square c_{i}: z \in V_{i j}\right\} .
$$

This decomposition corresponds to a diagonalization of the adjoint action of $\mathcal{A}$ since

$$
[H, X]=\frac{\lambda_{j}(H)-\lambda_{i}(H)}{2} X, X \in \mathcal{N}_{i j}
$$

Finally, let $V^{\mathbb{C}}=V+i V$ be the complexification of $V$. The action of $G$ is extended to $V^{\mathbb{C}}$ in the obvious way. 
3.2. Preliminaries on irreducible symmetric Siegel domains of tube type. We keep notations of the previous section and let

$$
\mathcal{D}=\left\{z \in V^{\mathbb{C}}: \operatorname{Im} z \in \Omega\right\} .
$$

The elements $x \in V$ and $s \in S_{0}$ act on $\mathcal{D}$ in the following way:

$$
x \cdot z=z+x \quad, \quad s \cdot z=s z .
$$

Both actions generate a solvable Lie group

$$
S=V S_{0}=V N_{0} A=N A,
$$

which identifies with a group of holomorphic automorphisms acting simply transitively on $\mathcal{D}$. The Lie algebra $\mathcal{S}$ of $S$ admits the decomposition

$$
\mathcal{S}=V \oplus \mathcal{S}_{0}=\left(\bigoplus_{i \leq j} V_{i j}\right) \oplus\left(\bigoplus_{i<j} \mathcal{N}_{i j}\right) \oplus \mathcal{A} .
$$

Moreover, by (3.4) and (3.6), one knows the adjoint action of elements $H \in \mathcal{A}$ :

$$
\begin{array}{lll}
{[H, X]=\frac{\lambda_{i}(H)+\lambda_{j}(H)}{2} X \quad \text { for } \quad} & X \in V_{i j}, \\
{[H, X]=\frac{\lambda_{j}(H)-\lambda_{i}(H)}{2} X} & \text { for } \quad X \in \mathcal{N}_{i j} .
\end{array}
$$

Since $S$ acts simply transitively on the domain $\mathcal{D}$, we may identify $S$ and $\mathcal{D}$. More precisely, we define

$$
\theta: S \ni s \mapsto \theta(s)=s \cdot \mathbf{e} \in \mathcal{D},
$$

where $\mathbf{e}$ is the point $(0, i e)$ in $\mathcal{S}$. The Lie algebra $\mathcal{S}$ is then identified with the tangent space of $\mathcal{D}$ at $\mathbf{e}$ using the differential $d \theta_{e}$. We identify e with the unit element of $S$. We transport both the Bergman metric $g$ and the complex structure $\mathcal{J}$ from $\mathcal{D}$ to $S$, where they become left-invariant tensor fields on $S$. We still write $\mathcal{J}$ for the complex structure on $S$. Moreover, the complexified tangent space $T_{\mathrm{e}}^{\mathbb{C}}$ is identified with the complexification of $\mathcal{S}$, which we denote by $\mathcal{S}^{\mathbb{C}}$. The decomposition $T_{\mathbf{e}}^{\mathbb{C}}=T_{\mathbf{e}}^{(1,0)} \oplus T_{\mathbf{e}}^{(0,1)}$ is transported into

$$
\mathcal{S}^{\mathbb{C}}=\mathcal{Q} \oplus \mathcal{P} .
$$

Elements of $\mathcal{S}^{\mathbb{C}}$ are identified with left invariant vector fields on $S$, and are called left invariant holomorphic vector fields when they belong to $\mathcal{Q}$. The Kählerian metric given by the Bergman metric can be seen as a Hermitian form $(\cdot, \cdot)$ on $\mathcal{Q}$, and orthonormality for left invariant holomorphic vector fields means orthonormality for the corresponding elements in $\mathcal{Q}$.

Now, we construct a suitable orthonormal basis of $\mathcal{Q}$. Let $\left\{e_{j k}^{\alpha}\right\}$ be the orthonormal basis of $V$ fixed in the previous subsection. For $j<k$ and $1 \leq \alpha \leq d$, we define $X_{j k}^{\alpha} \in V_{j k}$ and $Y_{j k}^{\alpha} \in \mathcal{N}_{j k}$ as the left-invariant vector fields on $S$ corresponding to $e_{j k}^{\alpha}$ and $2 e_{j k}^{\alpha} \square c_{j}$, respectively. For each $j$ we define $X_{j}$ and $H_{j}$ as the left-invariant vector fields on $S$ corresponding to $c_{j} \in V_{j j}$ and $L\left(c_{j}\right) \in \mathcal{A}$, respectively.

Finally, let

which means that

$$
Z_{j}=X_{j}-i H_{j} \quad, \quad Z_{j k}^{\alpha}=X_{j k}^{\alpha}-i Y_{j k}^{\alpha}
$$

$$
\mathcal{J}\left(X_{j}\right)=H_{j} \quad, \quad \mathcal{J}\left(X_{j k}^{\alpha}\right)=Y_{j k}^{\alpha} .
$$

The left invariant vector fields $Z_{j}$, for $j=1, \cdots, r$, and $Z_{j k}^{\alpha}$, for $j<k \leq r$ and $\alpha=1, \cdots, d$. constitute an orthonormal basis of $\mathcal{Q}$. 
Using $Z_{j}$ we can compute the so called strongly diagonal Hua operators i.e the operators defined by

$$
\mathbb{H}_{j} f=\left(\mathbb{H} f \cdot Z_{j}, Z_{j}\right) \quad, \quad j=1, \cdots, r .
$$

In terms of the basis $X_{j}, X_{j k}^{\alpha}, Y_{j k}^{\alpha}, H_{j}$ they are [BBDHPT]:

$$
\mathbb{H}_{j}=\Delta_{j}+\frac{1}{2} \sum_{k<j} \sum_{\alpha} \Delta_{k j}^{\alpha}+\frac{1}{2} \sum_{l>j} \sum_{\alpha} \Delta_{j l}^{\alpha},
$$

where

$$
\Delta_{j}=X_{j}^{2}+H_{j}^{2}-H_{j} \quad \Delta_{i j}^{\alpha}=\left(X_{i j}^{\alpha}\right)^{2}+\left(Y_{i j}^{\alpha}\right)^{2}-H_{j} .
$$

$\Delta_{j}$ 's and $\Delta_{i j}^{\alpha}$ 's are $S$-invariant operators, which at the point $i e$ agree with $\partial_{z_{j}} \partial_{\bar{z}_{j}}$ and $\partial_{z_{i j}^{\alpha}} \partial_{\bar{z}_{i j}^{\alpha}}$.

3.3. Further notations. In this subsection, we collect some information and some notations which will be used in the proof, in the next section. We assume that $r \geq 2$. We will define some sub-algebras and subgroups. Let

$$
\begin{array}{ccc}
\mathcal{A}^{-}=\operatorname{lin}\left\{L\left(c_{1}\right), \ldots, L\left(c_{r-1}\right)\right\}, & \mathcal{A}^{+}=\operatorname{lin}\left\{L\left(c_{r}\right)\right\}, \\
\mathcal{N}_{0}^{-}=\bigoplus_{i<j \leq r-1} \mathcal{N}_{i j} & \text { and } \quad \mathcal{N}_{0}^{+}=\bigoplus_{j=1}^{r-1} \mathcal{N}_{j r} .
\end{array}
$$

Then $\mathcal{N}_{0}^{+}$is an ideal of $\mathcal{N}_{0}$, while $\mathcal{N}_{0}^{-}$is a subalgebra. Clearly

$$
\mathcal{A}=\mathcal{A}^{-} \oplus \mathcal{A}^{+}
$$

and

$$
\mathcal{N}_{0}=\mathcal{N}_{0}^{-} \oplus \mathcal{N}_{0}^{+} .
$$

Next, we define $A^{+}, A^{-}, N_{0}^{+}, N_{0}^{-}$as the exponentials of the corresponding Lie algebras. We have

$$
A=A^{-} A^{+} \quad \text { and } \quad N_{0}=N_{0}^{-} N_{0}^{+}
$$

in the sense that the mappings

$$
\begin{aligned}
A^{-} \times A^{+} & \rightarrow A \\
\left(a^{-}, a^{+}\right) & \mapsto a^{-} a^{+}
\end{aligned} \quad \text { and } \quad \begin{aligned}
N_{0}^{-} \times N_{0}^{+} & \rightarrow N_{0} \\
\left(y^{-}, y^{+}\right) & \mapsto y^{-} y^{+}
\end{aligned}
$$

are diffeomorphisms.

3.4. Special coordinates. Let $\partial \mathcal{D}=V+i \partial \Omega$ be the topological boundary of the domain $\mathcal{D}=V+i \Omega$. We fix a Jordan frame, choose coordinates in $V$ according to the Peirce decomposition $x=\sum x_{j k}^{\alpha} e_{j k}^{\alpha}$ and we order them lexicographically i.e. $(j, k)>(l, p)$ if either $j>l$ or $j=l$ and $k>p$.

Let $S=V N_{0} A$ be the corresponding solvable Lie group. We consider its subgroup $S^{\prime}=V N_{0} A^{-}$ that will be identified with $\mathbb{R}^{2 n-1}$. To determine appropriate coordinates in $S^{\prime}$ we look more carefully at the transformations that build $N_{0}$. Namely [FK], any element of $N_{0}$ can be written uniquely as

$$
\tau\left(y^{1}\right) \cdot \ldots \cdot \tau\left(y^{r-1}\right),
$$

where $y^{j}=\sum_{j<k \leq r} y_{j k}^{\alpha} e_{j k}^{\alpha}$ and $\tau\left(y^{j}\right)=\exp \left(2 y^{j} \square c_{j}\right)$.

Therefore, any element $x \tau\left(y^{1}\right) \cdot \ldots \cdot \tau\left(y^{r-1}\right) \exp \left(\sum_{j<r} y_{j j} L\left(c_{j}\right)\right)$ of $S^{\prime}$ can be uniquely written as $\left(x, y_{11}, y^{1}, y_{22}, y^{2}, \ldots, y^{r-1}\right)$. Notice that the coordinates are ordered lexicographically exactly as in $\mathcal{D} \subset V+i V$. Now we define a diffeomorphism $\phi$ of $S^{\prime}=\mathbb{R}^{2 n-1}$ onto an open subset of $\partial \mathcal{D}$ as the limit point of the curve $t \mapsto s^{\prime} \exp \left(-t H_{r}\right) \cdot i e$ :

$$
\phi\left(s^{\prime}\right)=\lim _{t \rightarrow \infty} s^{\prime} \exp \left(-t H_{r}\right) \cdot i e .
$$


In the above coordinates we have

$$
\begin{aligned}
& \phi\left(x, y_{11}, y^{1}, y_{22}, y^{2}, \ldots, y_{r-1, r-1}, y^{r-1}\right)= \\
& \quad=x+i\left(\sum_{\substack{j<k \\
\alpha}}\left(e^{y_{j j}} y_{j k}^{\alpha}+P_{j k}^{\alpha}(y)\right) e_{j k}^{\alpha}+i \sum_{j<r}\left(e^{y_{j j}}+P_{j j}(y)\right) c_{j}+P_{r r}(y) c_{r}\right),
\end{aligned}
$$

where $P_{j k}^{\alpha}$ and $P_{j j}$ are polynomials depending only on the coordinates that proceed $y_{j k}^{\alpha}$ or $y_{j j}$ in the above order i.e. on $y_{l p}$ for $(l, p)<(j, k)$ or $(l, p)<(j, j)$, which follows from triangularity of the action of the group $N_{0}$, given by (3.5). To obtain explicit formulas for the polynomials one has to use a more precise formula for $\tau$, as described in Chapter VI of [FK], see also DHMP].

Lemma 3.1. The mapping $\phi$ is an one-to-one diffeormorphism of $\mathbb{R}^{2 n-1}$ onto an open subset of $\partial \mathcal{D}$.

Proof. One gets that $\phi$ is one-to-one from formula (3.14) and the observation that the action of $N$ is triangular. To prove that $\phi$ is a diffeomorphism it is enough to compute $d \phi$, the differential of $\phi$. Using again triangularity of the action of $N$, one can easily find a minor of rank $2 n-1$, which is a triangular matrix with 1's and $e^{y_{j j}}$ 's on the diagonal. The Inverse Mapping Theorem implies the claim.

Using $\phi$ we define a coordinate system on a neighborhood of $\phi\left(\mathbb{R}^{2 n-1}\right)$

$$
\mathbb{R}^{2 n-1} \times \mathbb{R} \ni(w, b) \mapsto \phi(w)+i b c_{r} \in V+i V,
$$

where $w=\left(x, y_{11}, y^{1}, y_{22}, y^{2}, \ldots, y_{r-1, r-1}, y^{r-1}\right)$ is identified with the corresponding element $s^{\prime}$ of the group $S^{\prime}$. This means that for positive $b$

$$
\phi(w)+i b c_{r}=s^{\prime} \exp \left(b L\left(c_{r}\right)\right) \cdot i e \in \mathcal{D}
$$

and

$$
\phi(w)+i b c_{r} \notin \overline{\mathcal{D}}
$$

if $b$ is negative. For every Jordan frame $c_{1}, \ldots, c_{r}$ and the corresponding group $S$ we may construct such a system. Moreover, applying an element $g$ of the group $G$ to it, we obtain a coordinate system on a neighborhood of $g\left(\phi\left(S^{\prime}\right)\right)$ satisfying (3.15) and (3.16). We are going to exploit such systems to define regularity of a function near the boundary of $\mathcal{D}$.

Definition. We say that a coordinate system $\Phi: \mathbb{R}^{2 n-1} \times \mathbb{R} \mapsto V+i V$ is a special coordinate system if it is of the form

$$
\Phi(w, b)=g(\phi(w))+i b g\left(c_{r}\right)
$$

for a Jordan frame $c_{1}, \ldots c_{r}$ and a $g \in G$.

Special coordinate systems are suitable to describe the boundary behavior of bounded pluriharmonic (holomorphic) functions, in terms of some integral conditions. More precisely, we consider functions satisfying the following regularity condition:

Condition 3.18. A function $F$ is said to satisfy Condition 3.18 for some integer $k$ if, for every special coordinate system $\Phi$, and every $\psi \in \mathcal{S}\left(\mathbb{R}^{2 n-1}\right)$,

$$
\sup _{0<b<1}\left|\int_{\mathbb{R}^{2 n-1}} \partial_{b}^{k} F(\Phi(w, b)) \psi(w) d w\right|<\infty .
$$

for some integer number $k$, where $d w$ is the Lebesgue measure.

Proposition 3.2. Let $F$ be a bounded pluriharmonic function on $\mathcal{D}$, then $F$ satisfies Condition 3.18 for every $k$. 
Proof. The action of $G$ preserves pluriharmonicity and $F(\Phi(w, b))=(F \circ g)\left(\phi(w)+i b c_{r}\right)$ for some $g \in G$. Notice also that if $F$ satisfies Condition 3.18 for some $k$, then so does $F_{g}(z)=F(g \cdot z)$. Therefore, we may assume that $g=I d$ in (3.17). Moreover, it is enough to prove (3.18) for even $k$ 's. Now since

$$
0=\partial_{z_{r}} \partial_{\bar{z}_{r}} F=\left(\partial_{x_{r}}^{2}+\partial_{b}^{2}\right) F
$$

we have

$$
\begin{aligned}
\int_{\mathbb{R}^{2 n-1}} \partial_{b}^{2 k} F\left(\phi(w)+i b c_{r}\right) \psi(w) d w & =\int_{\mathbb{R}^{2 n-1}}\left(-\partial_{x_{r}}^{2}\right)^{k} F\left(\phi(w)+i b c_{r}\right) \psi(w) d w \\
& =(-1)^{k} \int_{\mathbb{R}^{2 n-1}} F\left(\phi(w)+i b c_{r}\right)\left(\partial_{x_{r}}^{2}\right)^{k} \psi(w) d w
\end{aligned}
$$

and (3.18) follows.

3.5. Maximum regularity of Hua-harmonic functions. Now we are in position to formulate and to prove the main result of the paper

Main Theorem 3.3. Let $\Omega$ be an irreducible symmetric cone if rank $r$ and let $d$ be the common dimension of the associated spaces in its Peirce decomposition (3.3). Let $\mathcal{D}$ be the tube type domain associated to $\Omega$ If $F$ is a bounded Hua-harmonic function on $\mathcal{D}$ satisfying Condition $3.1 \mathrm{~d}$ for $k=$ $0, \ldots,\left[\frac{(r-1) d+1}{2}\right]+1$, then $F$ is pluriharmonic.

Remark. Notice that $F$ in the above theorem has certain boundary regularity at any point $z \in \partial \mathcal{D}$ for which $\operatorname{Im} z$ has rank $r-1$ in the Jordan algebra. The set of such points will be denoted $\widetilde{\partial \mathcal{D}}$. The theorem says that this regularity implies pluriharmonicity.

Fix a Jordan frame $c_{1}, \ldots, c_{r}$, the corresponding group $S$ and differential operators $\Delta_{k}, \Delta_{j k}^{\alpha}$, (3.13). We will show that

$$
\begin{aligned}
\Delta_{k} F=0 & \text { for } k=1, \ldots, r \\
\Delta_{j k}^{\alpha} F=0 & \text { for } 1 \leq j<k \ldots, r \text { and } \alpha=1, \ldots, d
\end{aligned}
$$

and the conclusion will follow by $\mathrm{BDH}$.

Let

Then clearly,

$$
N^{+}=V^{+} N_{0}^{+}, \quad N^{-}=V^{-} N_{0}^{-} .
$$

$$
N=N^{-} N^{+}, \quad S^{-}=N^{-} A^{-}, \quad S^{+}=N^{+} A^{+} .
$$

Denote by $d n^{-}, d n^{+}, d a^{-}, d a^{+}$the Haar measures on the groups $N^{-}, N^{+}, A^{-}, A^{+}$respectively and $d s^{-}=d n^{-} d a^{-}, d s^{+}=d n^{+} d a^{+}$.

Notice that $N^{+}$is the Heisenberg group and $S^{+}$is one parameter extension considered in Section 2. Indeed, for the basis $X_{j r}^{\alpha}, Y_{j r}^{\alpha}, X_{r}, H_{r}$ of the Lie algebra of $S^{+}$we have

$$
\begin{aligned}
{\left[Y_{j r}^{\alpha}, X_{j r}^{\alpha}\right] } & =X_{r}, & {\left[H_{r}, X_{r}\right] } & =X_{r}, \\
{\left[H_{r}, X_{j r}^{\alpha}\right] } & =\frac{1}{2} X_{j r}^{\alpha}, & {\left[H_{r}, Y_{j r}^{\alpha}\right] } & =\frac{1}{2} Y_{j r}^{\alpha},
\end{aligned}
$$

while all other brackets are 0 (see (1.16) and (1.17) of DHMP).

Given $\psi \in \mathcal{C}_{c}^{\infty}\left(S^{-}\right)$, let

$$
G_{\psi}\left(s^{+}\right)=\int_{S^{-}} F\left(s^{-} s^{+}\right) \psi\left(s^{-}\right) d s^{-} .
$$

The operator $\mathbb{H}_{r}$ is well defined for a function on $S^{+}$and

$$
\mathbb{H}_{r} G_{\psi}\left(s^{+}\right)=\int_{S^{-}}\left(\mathbb{H}_{r} F\right)\left(s^{-} s^{+}\right) \psi\left(s^{-}\right) d s^{-} .
$$


Therefore

$$
\mathbb{H}_{r} G_{\psi}=0 \text {. }
$$

Notice that $\mathbb{H}_{r}$ is the operator $L_{1 / 2}$ from Section 2 .

Lemma 3.4. Assume that $F$ satisfies the conditions of Theorem 3.3. Then, for every $\phi \in \mathcal{S}\left(N^{+}\right)$ and every $k=1, \ldots,\left[\frac{(r-1) d+1}{2}\right]+1$,

$$
\sup _{0<a^{+}<1}\left|\int_{N^{+}} \partial_{a^{+}}^{k} G_{\psi}\left(n^{+} a^{+}\right) \phi\left(n^{+}\right) d n^{+}\right|<\infty .
$$

Proof. Define

so that

$$
I\left(a^{+}\right)=\int_{N^{+}} G_{\psi}\left(n^{+} a^{+}\right) \phi\left(n^{+}\right) d n^{+}
$$

$$
\begin{aligned}
I\left(a^{+}\right) & =\int_{N^{+}} \int_{S^{-}} F\left(s^{-} n^{+} a^{+}\right) \psi\left(s^{-}\right) \phi\left(n^{+}\right) d s^{-} d n^{+} \\
& =\int_{N^{+}} \int_{S^{-}} F\left(n^{-} a^{-} n^{+} a^{+}\right) \psi\left(s^{-}\right) \phi\left(n^{+}\right) d n^{-} d a^{-} d n^{+} .
\end{aligned}
$$

Now we change variables via the transformation

$$
n^{+} \mapsto\left(a^{-}\right)^{-1} n^{+} a^{-} .
$$

Then

$$
\begin{aligned}
I\left(a^{+}\right) & =\int_{S^{-}} \int_{N^{+}} F\left(n^{-} n^{+} a^{-} a^{+}\right) \psi\left(s^{-}\right) \phi\left(\left(a^{-}\right)^{-1} n^{+} a^{-}\right) \prod_{j=1}^{r-1} a_{j}^{-\frac{d}{2}} d n^{+} d n^{-} d a^{-} \\
& =\int_{\mathbb{R}^{2 n-1}} F\left(\Phi\left(w, a^{+}\right)\right) \widetilde{\psi}(w) d w
\end{aligned}
$$

for some $\widetilde{\psi}$ in the Schwartz class. So (3.21) follows from (3.18).

Lemma 3.5. Assume that $F$ satisfies the conditions of Theorem 3.3, then

$$
\Delta_{r} F=0 \quad \text { and } \quad \Delta_{j r}^{\alpha} F=0
$$

for $j=1, \ldots, r-1$ and $\alpha=1, \ldots, d$.

Proof. Let $\psi \in \mathcal{S}\left(S^{-}\right)$. By Theorem 2.7, the function $G_{\psi}$ is pluriharmonic and so $\Delta_{r} G_{\psi}=0$ and for $j=1, \ldots, r, \alpha=1, \ldots, d$, we have $\Delta_{j r}^{\alpha} G_{\psi}=0$. But

$$
\Delta_{j r}^{\alpha} G_{\psi}\left(s^{+}\right)=\int_{S^{-}} \Delta_{j r}^{\alpha} F\left(s^{-} s^{+}\right) \psi\left(s^{-}\right) d s^{-}, \quad \Delta_{r} G_{\psi}\left(s^{+}\right)=\int_{S^{-}} \Delta_{r} F\left(s^{-} s^{+}\right) \psi\left(s^{-}\right) d s^{-}
$$

for any $\psi$, so the conclusion follows.

End of the proof of Theorem 3.3. We have just proved

$$
\begin{aligned}
\partial_{z_{j r}^{\alpha}} \partial_{\bar{z}_{j r}^{\alpha}} F(e) & =0 \quad \text { for } j \leq r-1, \text { and } \alpha=1, \ldots, d \\
\partial_{z_{r}} \partial_{\bar{z}_{r}} F(e) & =0 .
\end{aligned}
$$

Taking any element $k \in \operatorname{Aut}(V)$ permuting the chosen Jordan frame and repeating the above argument, but with the group $S_{k}=k S k^{-1}$ instead of $S$, we may prove that $F$ is annihilated by the $S_{k}$-invariant operators $k \Delta_{j r}^{\alpha} k^{-1}$. Therefore,

$$
\begin{aligned}
\partial_{z_{j k}^{\alpha}} \partial_{\bar{z}_{j k}^{\alpha}} F(e)=0 & \text { for } j<k \leq r, \text { and } \alpha=1, \ldots, d \\
\partial_{z_{k}} \partial_{\bar{z}_{k}} F(e)=0 & \text { for } k \leq r
\end{aligned}
$$


Take any $s \in S$, then $F_{s}=F \circ s$ satisfies assumptions of Theorem 3.3, hence

$$
\begin{array}{cl}
\partial_{z_{j k}^{\alpha}} \partial_{\bar{z}_{j k}^{\alpha}}(F \circ s)(e)=0 & \text { for } j<k \leq r, \text { and } \alpha=1, \ldots, d \\
\partial_{z_{k}} \partial_{\bar{z}_{k}}(F \circ s)(e)=0 & \text { for } k \leq r
\end{array}
$$

which implies (3.19) and completes the proof of the main theorem.

\section{Appendix A. A lemma about Confluent hypergeometric equations}

Notation. For $a \in \mathbb{R}$ and $n \in \mathbb{N}$, we denote by $(a)_{0}=1,(a)_{1}=a$ and $(a)_{n}=a(a+1) \ldots(a+n-1)$. Further, if $a, c \in \mathbb{R}$ and $c \notin \mathbb{Z}^{-}$, then

$$
{ }_{1} F_{1}(a, c, x)=\sum_{n=0}^{+\infty} \frac{(a)_{n}}{(c)_{n}} \frac{x^{n}}{n !}
$$

defines an entire function (called the confluent hypergeometric function) that satisfies the following differential equation:

$$
x y^{\prime \prime}+(c-x) y^{\prime}-a y=0 .
$$

Notice that, for $x>0$, if $a>c>0$, then ${ }_{1} F_{1}(a, c, x) \geq e^{x}$, whereas if $a>0>c$, then ${ }_{1} F_{1}(a, c, x) \geq$ $e^{x}-P(x)$ where $P$ is a polynomial.

We are interested in bounded solutions on $] 0,+\infty)$ of a variant of this equation.

Lemma A.1. Let $\gamma>0$ and $\beta \geq 0$ and let $k$ be the smallest integer greater than $\gamma$, that is $k=[\gamma]+1$ if $\gamma$ is not an integer and $k=\gamma$ otherwise. Then, the equation

$$
x g^{\prime \prime}-\gamma g^{\prime}-(x+(\gamma+\beta)) g=0
$$

has one bounded non zero solution $y$ on $(0,+\infty)$ and only one, up to a constant. For $\beta \neq 0$, its derivatives have finite limits at 0 up to the order $k$. Moreover, for every positive integer $N$, there exists two functions, $a_{1}$ and $a_{2}$, which are smooth up to order $N$ on $[0, \infty)$, such that $a_{1}(0) \neq 0$ and

$$
\partial^{k+1} y(x)=\left\{\begin{array}{ll}
a_{1}(x) \ln x+a_{2}(x) & \text { if } \gamma \text { is an integer } \\
a_{1}(x) x^{\gamma-k}+a_{2}(x) & \text { if } \gamma \text { is not an integer }
\end{array} .\right.
$$

For $\beta=0$, the solution $y$ is given by $y(x)=e^{-x}$ and is an entire function.

Proof. We leave the computations for the reader when $\beta=0$ since they are completely elementary.

¿From now on, we assume that $\beta>0$. Let us look for solutions of $(\mathrm{A} .26)$ of the form $g(x)=$ $e^{-x} h(2 x)$. Then $h$ is a solution of the equation

$$
x h^{\prime \prime}-(x+\gamma) h^{\prime}-\left(\frac{\beta}{2}\right) h=0 .
$$

When $\gamma$ is not a non negative integer, the function ${ }_{1} F_{1}\left(\frac{\beta}{2},-\gamma, \cdot\right)$ is a solution of (A.27). Moreover, it increases at infinity in such a way that the corresponding solution of (A.26) is unbounded. For $\gamma$ a non negative integer, there is still an entire function which is a solution of the equation A.27) and gives rise to an unbounded solution of (A.26). Coefficients of its Taylor series vanish up to $-c$. The uniqueness, up to a constant, of bounded solutions of (A.26) on $] 0, \infty$ ) follows at once since the vector space of solutions, which is of dimension 2 , can only have a proper subspace of dimension 1 of bounded solutions.

Let us now show the existence of a bounded solution on $] 0,+\infty)$ as well as the asymptotic behavior of the $(k+1)$-th derivative. Using a Laplace transform for (A.27), it is easy to propose another solution for $(\mathrm{A} .26)$,

$$
y(x)=e^{-x} \int_{0}^{\infty} e^{-2 x t} \frac{t^{\frac{\beta}{2}-1}}{(1+t)^{\gamma+\frac{\beta}{2}}} d t .
$$


It is easily seen that $y$ is a $\mathcal{C}^{\infty}$ function on $(0,+\infty)$, of class $\mathcal{C}^{k}$ on $[0,+\infty)$, and bounded. It remains to find the explicit expression for the derivative of order $k+1$ of $y$. Let us first assume that $\gamma$ is not an integer. We look for another solution of A.26 which may be written as $x^{\gamma+1} e^{-x} h(2 x)$. Then $h$ is a solution of

$$
x h^{\prime \prime}-(x+\gamma+2) h^{\prime}-\left(\gamma+\frac{\beta+1}{2}\right) h=0 .
$$

So $y$, as a solution of (A.26), may be written

$$
y(x)=\alpha x^{\gamma+1} e^{-x}{ }_{1} F_{1}\left(\gamma+\frac{\beta+1}{2}, \gamma+2,2 x\right)+\tilde{\alpha} e^{-x}{ }_{1} F_{1}\left(\frac{\beta}{2},-\gamma, 2 x\right) .
$$

Moreover $\alpha \neq 0$ since $y$ is bounded. This allows to conclude in this case, taking derivatives up to order $k+1$.

Let us now consider the case when $\gamma=k-1$ is an integer. We will use the explicit formula of $y$ as an integral. Moreover, cutting the integral into two, from 0 to 1 and from 1 to infinity, we see that the first integral is a $\mathcal{C}^{\infty}$ function. So it is sufficient to consider

$$
\int_{1}^{\infty} e^{-2 x t} \frac{t^{\frac{\beta}{2}-1}}{(1+t)^{k+\frac{\beta}{2}}} d t=\int_{1}^{\infty} e^{-2 x t} t^{-k}\left(1+\frac{1}{t}\right)^{k-1+\frac{\beta}{2}} d t
$$

Moreover, taking the Taylor expansion of the term $\left(1+\frac{1}{t}\right)^{k-1+\frac{\beta}{2}}$, it is sufficient to consider terms up to order $N$, the remaining part giving rise to a $\mathcal{C}^{N+k}$ function. Finally, after having taken derivatives, it is sufficient to prove that each term

$$
I_{j}(x):=\int_{1}^{\infty} e^{-x t} t^{-j-1} d t
$$

may be written as $\alpha_{j} x^{j} \ln x+a_{j}(x)$, with $\alpha_{0} \neq 0$ and $a_{j}$ a $\mathcal{C}^{\infty}$ function. Integration by parts allows to reduce to the case when $j=0$. But a direct computation gives

$$
I_{0}(x)=\int_{1}^{\infty} e^{-t} \frac{d t}{t}+\sum_{l=0}^{\infty} \frac{(-1)^{\ell}}{\ell !} \int_{x}^{1} t^{l-1} d t,
$$

from which we conclude easily.

\section{Appendix B. A lemma about Legendre equations}

Notation. For $c \in \mathbb{R} \backslash \mathbb{Z}^{-}$, define the entire function

$$
{ }_{0} F_{1}(c, x)=\sum_{k=0}^{\infty} \frac{1}{(c)_{k}} \frac{x^{k}}{k !}
$$

defines an entire function, called the Legendre function, that satisfies the following differential equation:

$$
x y^{\prime \prime}(x)+c y^{\prime}(x)-y(x)=0 .
$$

For $c$ a negative integer, there is still an entire function which is a solution of the Legendre equation. Coefficients of its Taylor series vanish up to $-c$.

We are interested in bounded solutions of the Legendre equation for negative values of the parameter.

Lemma B.1. Let $\beta>0$ and let $k$ be the smallest integer greater than $\beta$. Then the equation

$$
x g^{\prime \prime}-\beta g^{\prime}-g=0
$$


has a bounded non zero solution $y$ on $] 0,+\infty)$ whose derivatives have finite limits at 0 up to the order $k$. For every positive integer $N$, there exists two functions, $a_{1}$ and $a_{2}$, which are smooth up to order $N$ on $[0, \infty)$, such that $a_{1}(0) \neq 0$ and

$$
\partial^{k+1} y(x)= \begin{cases}a_{1}(x) \ln x+a_{2}(x) & \text { if } \beta \text { is an integer } \\ a_{1}(x) x^{\beta-k}+a_{2}(x) & \text { if } \beta \text { is not an integer }\end{cases}
$$

Moreover, all non zero bounded solutions of (B.28) behave as above.

Proof. The uniqueness, up to a constant, of bounded solutions follow from the fact that the entire functions that are solutions increase faster than any polynomial at infinity. So the vector space of solutions, which is of dimension 2, can only have a proper subspace of dimension 1 of bounded solutions. Let us now show the existence of a bounded solution on $(0,+\infty)$.

This one is given by the Laplace method:

$$
y(x)=\int_{0}^{+\infty} \frac{e^{-x t} e^{-1 / t}}{t^{2+\beta}} d t .
$$

It is clear that $y$ is rapidly decreasing and has derivatives up to order $k$ at 0 . It remains to find the explicit formula for the $k+1$-th derivative. When $\beta$ is not an integer, we look for another solution of (B.28) which may be written as $x^{\beta+1} h(x)$. Then $h$ is a solution of the equation

$$
x h^{\prime \prime}+(\beta+2) h^{\prime}-1=0 .
$$

As a consequence, the function $y$, which is a solution of (B.28), may be written as

$$
y(x)=\alpha x_{0}^{\beta+1} F_{1}(\beta+2, x)+\tilde{\alpha}_{0} F_{1}(-\beta, x),
$$

with $\alpha \neq 0$ since ${ }_{0} F_{1}(-\beta, \cdot)$ is not bounded. This allows to conclude in this case, taking derivatives up to order $k+1$.

For $\beta=k$, we use the explicit formula for the function $y$, that is, up to the constant $(-1)^{k+1}$,

$$
\int_{0}^{1} \frac{e^{-x t} e^{-1 / t}}{t} d t+\int_{1}^{+\infty} \frac{e^{-x t}\left(e^{-1 / t}-\sum_{j=0}^{N}(-1 / t)^{j} / j !\right)}{t} d t+\sum_{j=0}^{N} c_{j} \int_{1}^{+\infty} \frac{e^{-x t}}{t^{j+1}} d t
$$

with $c_{j}=(-1)^{j} / j$ !. Now, the two first integrals are smooth up to order $N$. It remains to consider each term of the last sum, for which we conclude as in the previous section.

\section{Appendix C. Some complements on Harmonic Analysis on the Heisenberg group}

C.1. A lemma about Laguerre functions. We come back to the notations of Section 2.4. Further lemmas we may need can be found e.g. in [1].

Recall that we defined a unitary representation $R^{\lambda}$ of $\mathbf{H}^{n}$ on $L^{2}\left(\mathbb{R}^{n}\right)$ by

$$
R^{\lambda}(\zeta, t) \Phi(x)=e^{2 \pi i \lambda(u \cdot x+u \cdot v / 2+t / 4)} \Phi(x+v)
$$

The Hermite functions $H_{k}$ of one variable are defined by the formula

$$
h_{k}(x)=(-1)^{k} c_{k} e^{x^{2} / 2}\left(\frac{d}{d x}\right)^{k} e^{-x^{2}}, \quad k=0,1,2, \ldots,
$$

with $c_{k}=\left(\sqrt{\pi} 2^{k} k !\right)^{1 / 2}$. For $k=\left(k_{1}, \ldots, k_{n}\right)$ a multi-index, we write $h_{k}=h_{k_{1}} \ldots h_{k_{n}}$.

Now, for $\lambda \neq 0$, denote by $h_{k}^{\lambda}(x)=(2 \pi|\lambda|)^{n / 4} h_{k}\left((2 \pi|\lambda|)^{1 / 2} x\right)$. Finally, let

$$
e_{\kappa}^{\lambda}(\omega)=\sum_{|k|=\kappa}\left(R^{\lambda}(\omega) h_{k}^{\lambda}, h_{k}^{\lambda}\right)
$$


and, for $\psi \in C_{c}^{\infty}(\mathbb{R} \backslash\{0\})$,

$$
e_{k}^{\psi}(\zeta, t)=\int_{\mathbb{R}} e_{k}^{\lambda}(\zeta, t) \psi(\lambda) d \lambda
$$

Then, $e_{k}^{\phi} \in \mathcal{S}\left(\mathbb{C}^{n} \times \mathbb{R}\right)$.

Lemma C.1. Let $\varepsilon>0$. There exists constants $C, M>0$ such that, for every $\psi \in C_{c}^{\infty}(\mathbb{R})$ with $\operatorname{supp} \psi \subset\left[\varepsilon, \varepsilon^{-1}\right]$, and every $\kappa \in \mathbb{N}$,

$$
\left\|e_{\kappa}^{\psi}\right\|_{L^{1}} \leq C \kappa^{M}\left(\|\psi\|_{L^{\infty}}+\left\|\psi^{\prime}\right\|_{L^{\infty}}\right)
$$

Before proving this lemma we need more information about the function $\Phi_{k}=\left\langle R^{\lambda} h_{k}^{\lambda}, h_{k}^{\lambda}\right\rangle$. Let $L_{k}$ be the $k$-th Laguerre polynomial, i.e.

$$
L_{k}(t) e^{-t}=\frac{1}{k !}\left(\frac{d}{d t}\right)^{k}\left(e^{-t} t^{k}\right) .
$$

Given a multi-index $k=\left(k_{1}, \ldots, k_{n}\right)$ and $\zeta \in \mathbb{C}^{n}$ let

$$
\mathrm{L}_{k}(\zeta)=L_{k_{1}}\left(\frac{1}{2}\left|\zeta_{1}\right|^{2}\right) \times \ldots \times L_{k_{n}}\left(\frac{1}{2}\left|\zeta_{n}\right|^{2}\right) .
$$

Then

$$
\Phi_{k}(\zeta)=(2 \pi)^{-n / 2} \mathrm{~L}_{k}(\zeta) e^{-\frac{1}{4}|\zeta|^{2}},
$$

(see Formula (1.4.20) [1])

We will use the following well-known property of the Laguerre functions (see BDH).

Lemma C.2. For every $l, p \in \mathbb{N}$ there exist $c=c(l, p)$ and $M=M(l, p)$ such that, for every $k \in \mathbb{N}$,

$$
\int_{0}^{\infty} t^{l}\left|\partial_{t}^{p} L_{k}(t)\right|^{2} e^{-t} d t \leq c k^{M}
$$

Proof of lemma C.1. To estimate the $L^{1}\left(\mathbf{H}^{n}\right)$ norm of $e_{\kappa}^{\psi}$, we first use Schwartz' inequality to see that it is sufficient to have a bound for

$$
I_{k}:=\int_{\mathbf{H}^{n}}\left(1+t^{2}\right)\left(1+\left|\zeta_{1}\right|^{4}\right) \times\left(1+\left|\zeta_{2}\right|^{4}\right) \times \cdots \times\left(1+\left|\zeta_{n}\right|^{4}\right) \times\left|\phi_{k}(\zeta, t)\right|^{2} d t d \zeta
$$

where

$$
\phi_{k}(\zeta, t):=\int_{\mathbb{R}} e^{i \lambda t} \psi(\lambda) \Phi_{k}(\sqrt{\lambda} \zeta) d \lambda
$$

We use Parseval Identity in the $t$ variable to write that

$$
I_{k}=\int_{\mathbb{C}^{n}} \int_{\mathbb{R}}\left(1+\left|\zeta_{1}\right|^{4}\right) \times \cdots \times\left(1+\left|\zeta_{n}\right|^{4}\right) \times\left(\left|\psi(\lambda) \Phi_{k}(\sqrt{\lambda} \zeta)\right|^{2}+\left|\partial_{\lambda}\left\{\psi(\lambda) \Phi_{k}(\sqrt{\lambda} \zeta)\right\}\right|^{2}\right) d \lambda d \zeta .
$$

We find that $I_{k} \leq C J(k)\left(\|\psi\|_{L^{\infty}}+\left\|\psi^{\prime}\right\|_{L^{\infty}}\right)^{2}$, with

$$
J(k):=\int_{\mathbb{C}^{n}} \int_{\mathbb{R}}\left(1+\left|\zeta_{1}\right|^{4}\right) \times \cdots \times\left(1+\left|\zeta_{n}\right|^{4}\right) \times\left(\left|\Phi_{\alpha, \alpha}(\sqrt{\lambda} \zeta)\right|^{2}+\left|\partial_{\lambda}\left\{\Phi_{\alpha, \alpha}(\sqrt{\lambda} \zeta)\right\}\right|^{2}\right) d \lambda d \zeta .
$$

Now we use Relation (C.32), and integrate first in $\zeta$, using Lemma C.2. We then find that $J(k) \leq c \kappa^{M}$ where $\kappa=|k|$. We have thus proved (C.30). 
C.2. The Fourier Inversion Formula. In this section, we adapt the Fourier Inversion Formula to our choice of representation of the Heisenberg group. We only give an outline of the proofs, details with a similar choice of representation may be found in [Fo], pages 35-37.

The Fourier transform of an integrable function $f$ on $\mathbf{H}^{n}$ is the operator-valued function $\lambda \mapsto \hat{f}(\lambda)$ given by

$$
\langle\hat{f}(\lambda) \phi, \psi\rangle=\int_{\mathbf{H}^{n}}\left\langle R^{\lambda}(\mathrm{w}) \phi, \psi\right\rangle f(\mathrm{w}) d \mathrm{w} d t .
$$

To avoid any technicality, let us first assume that $f$ is bounded and compactly supported. One may then show that

$$
\operatorname{tr}\left(R^{\lambda}(\zeta, t) \widehat{f}(\lambda)\right)=|\lambda|^{-n} \mathcal{F}_{3} f(\zeta, \lambda / 4) e^{2 i \pi \lambda t / 4}
$$

where $\mathcal{F}_{3}$ is the partial Fourier transform in the $t$-variable. The ordinary Fourier Inversion Formula (in the $t$-variable) then leads to the Heisenberg Fourier Inversion Formula

$$
f(\zeta, t)=c \int_{\mathbb{R}} \operatorname{tr}\left(R^{\lambda}(\zeta, t) \widehat{f}(\lambda)\right)|\lambda|^{n} d \lambda .
$$

Now, let $\varphi \in \mathcal{S}(\mathbb{R})$ be such that $\widehat{\varphi}$ is compactly supported away from 0 and apply (C.35) to $f *_{3} \varphi$, the convolution in the $t$-variable of $f$ and $\varphi$, to get

$$
f *_{3} \varphi(\omega)=c \int_{\mathbb{R}} \operatorname{tr}\left(R^{\lambda}(\omega) \widehat{f *_{3} \varphi}(\lambda)\right)|\lambda|^{n} d \lambda,
$$

and, using twice (C.34), this becomes

$$
f *_{3} \varphi(\omega)=c \int_{\mathbb{R}} \operatorname{tr}\left(R^{\lambda}(\omega) \widehat{f}(\lambda)\right) \widehat{\varphi}(\lambda / 4)|\lambda|^{n} d \lambda .
$$

Next, rewriting the trace using the Hermite basis leads to

$$
\begin{aligned}
\operatorname{tr}\left(R^{\lambda}(\omega) \widehat{f}(\lambda)\right) & =\sum_{\kappa \in \mathbb{N}} \sum_{k:|k|=\kappa}\left\langle R^{\lambda}(\omega) \widehat{f}(\lambda) h_{k}^{\lambda}, h_{k}^{\lambda}\right\rangle \\
& =\sum_{\kappa \in \mathbb{N}} \sum_{k:|k|=\kappa} \int_{\mathbf{H}^{n}}\left\langle R^{\lambda}(\omega) R^{\lambda}(\eta, s) h_{k}^{\lambda}, h_{k}^{\lambda}\right\rangle f(\mathrm{w}) d \mathrm{w} \\
& =\sum_{\kappa \in \mathbb{N}} \int_{\mathbf{H}^{n}} e_{\kappa}^{\lambda}(\omega \mathrm{w}) f(\mathrm{w}) d \mathrm{w}=\sum_{\kappa \in \mathbb{N}} \int_{\mathbf{H}^{n}} e_{\kappa}^{\lambda}(\mathrm{w}) f\left(\omega^{-1} \mathrm{w}\right) d \mathrm{w} .
\end{aligned}
$$

Further, inserting this in (C.36), inverting summations and integrations, justified by Lemma C.1 and Fubini's theorem, we get

$$
f *_{3} \varphi(\omega)=\sum_{\kappa \in \mathbb{N}} \int_{\mathbf{H}^{n}} f\left(\omega^{-1} \mathrm{w}\right) e_{\kappa}^{\psi}(\mathrm{w}) d \mathrm{w}
$$

where $\psi(\lambda)=\widehat{\varphi}(\lambda / 4)|\lambda|^{n}$. Finally, note that Identity (C.37) is also valid for $f \in L^{\infty}$ since we may apply it to a truncation $f_{R}(\omega)=\left\{\begin{array}{ll}f(\omega) & \text { if }|\omega| \leq R \\ 0 & \text { else }\end{array}\right.$ and then let $R \rightarrow \infty$, again with the help of Lemma C.1. 


\section{Appendix D. Second Regularity CONDition}

In this section we show another regularity condition that leads to the pluriharmonicity of $F$. Since it is difficult to compare with the previous one, we include it here. Compared to the previous one, the conditions are given using only one solvable group $S$ and one special coordinate system.

We fix a Jordan frame $c_{1}, \ldots, c_{r}$ and the group $S$. Let

$$
\begin{aligned}
\mathcal{N}_{k}^{-}=\bigoplus_{i \leq j \leq k} V_{i j} \oplus \bigoplus_{i<j \leq k} \mathcal{N}_{i j}, & \mathcal{N}_{k}^{+}=\bigoplus_{k<i \leq j} V_{i j} \oplus \bigoplus_{k<i<j} \mathcal{N}_{i j}, \\
\mathcal{A}_{k}^{-}=\operatorname{lin}\left\{L\left(c_{1}\right), \ldots, L\left(c_{k}\right)\right\}, & \mathcal{A}_{k}^{+}=\operatorname{lin}\left\{L\left(c_{k+1}\right), \ldots, L\left(c_{r}\right)\right\} .
\end{aligned}
$$

Then $\mathcal{N}_{k}^{+}$is an ideal. Clearly

$$
\mathcal{N}=\mathcal{N}_{k}^{-} \oplus \mathcal{N}_{k}^{+}, \quad \mathcal{A}=\mathcal{A}_{k}^{-} \oplus \mathcal{A}_{k}^{+}
$$

Let

$$
\begin{array}{cl}
N_{k}^{-}=\exp \mathcal{N}_{k}^{-}, & N_{k}^{+}=\exp \mathcal{N}_{k}^{+}, \\
A_{k}^{-}=\exp \mathcal{A}_{k}^{-}, & A_{k}^{+}=\exp \mathcal{A}_{k}^{+}, \\
S_{k}^{-}=N_{k}^{-} A_{k}^{-}, & S_{k}^{+}=N_{k}^{+} A_{k}^{+} .
\end{array}
$$

Then

$$
N=N_{k}^{-} N_{k}^{+}, \quad S=S_{k}^{-} S_{k}^{+}
$$

in the sense that

$$
\begin{gathered}
S_{k}^{-} \times S_{k}^{+} \ni\left(s^{-}, s^{+}\right) \mapsto s^{-} s^{+} \in S, \\
N_{k}^{-} \times N_{k}^{+} \ni\left(n^{-}, n^{+}\right) \mapsto n^{-} n^{+} \in N
\end{gathered}
$$

are diffeomorphisms.

Let

$$
V_{k}^{-}=\bigoplus_{i \leq j \leq k} V_{i j}
$$

$V_{k}^{-}$is the Jordan algebra with the Jordan frame $c_{1}, \ldots, c_{r}$ and $S_{k}^{-}$is the solvable group acting simply transitively on $V_{k}^{-}+i \Omega_{k}^{-}$, where $\Omega_{k}^{-}=\operatorname{int}\left\{x^{2}: x \in V_{k}^{-}\right\}$. For every $k=1, \ldots r-1$ we define a subdomain

$$
\mathcal{D}_{k}=\mathcal{D} \cap\left\{z_{l j}=0, z_{j j}=i, \quad l<j, j>k\right\} .
$$

Clearly

$$
\mathcal{D}_{k}=S_{k}^{-} \cdot i e
$$

and

$$
\partial \mathcal{D}_{k}=\partial \mathcal{D} \cap\left\{z_{l j}=0, z_{j j}=i, \quad l<j, j>k\right\} .
$$

$\mathcal{D}_{k}$ is a symmetric tube domain corresponding to the cone $\Omega_{k}^{-}$.

Definition. Given a tube domain $\mathcal{D}$ with a fixed Jordan frame $c_{1}, \ldots, c_{r}$, the group $S$ and its subgroup $S^{\prime}$, we say that a bounded function is weak regular if it satisfies (3.18) with

$$
\Phi(w, b)=\phi(w)+i b c_{r}
$$

and $k=1, \ldots,\left[\frac{(r-1) d+1}{2}\right]+1$. 
It follows from a theorem by Lassalle $\left[\right.$ ] that $\phi\left(\mathbb{R}^{2 n-1}\right)$ is dense in $\partial \mathcal{D}$ although smaller than $\widetilde{\partial \mathcal{D}}$. Therefore, weak regularity means certain boundary regularity at any point from a dense subset of $\partial \mathcal{D}$. We are going to assume that the function is weak regular on the domain $\mathcal{D}$ and subdomains $\mathcal{D}_{k}$. More precisely, given $F$ we write a number of functions $K_{\psi}$ on the domains $\mathcal{D}_{k}$ :

$$
K_{\psi}(z)=\int_{S_{k}^{+}} F\left(s^{+} \cdot z\right) \psi\left(s^{+}\right) d s^{+},
$$

$\psi \in C_{C}^{\infty}\left(S_{k}^{+}\right), z \in \mathcal{D}_{k} . K_{\psi}$ will be identified with its version on the group $S_{k}^{-}$:

$$
K_{\psi}\left(s^{-}\right)=\int_{S_{k}^{+}} F\left(s^{+} s^{-} \cdot i e\right) \psi\left(s^{+}\right) d s^{+} .
$$

Definition. We say that $F$ is regular if $F$ is weak regular and for every $k=1, \ldots r-1$ and $\psi \in C_{C}^{\infty}\left(S_{k}^{+}\right)$, $K_{\psi}$ is weak regular on $\mathcal{D}_{k}$ with respect to the Jordan frame $c_{1}, \ldots c_{k}$.

Theorem D.1. Assume that $F$ is a real, bounded, Hua-harmonic and regular. Then $F$ is pluriharmonic.

Proof. We are going to prove by induction (3.19), which again, in view of [BDH], implies pluriharmonicity of $F$. First we notice that weak regularity of $F$ is sufficient to conclude that

$$
\Delta_{r} F=0 \text { and } \Delta_{j r}^{\alpha} F=0
$$

(see the proof of Lemma (3.5)). Hence for $j=r$ the conclusion holds.

Assume now that $\Delta_{i j}^{\alpha} F=0, \Delta_{j j} F=0$ for $j>k$ and $i<j$. We consider the operators

$$
\mathbb{H}_{j}^{k}=\Delta_{j j}+\frac{1}{2}\left(\sum_{\substack{i<j \\ \alpha}} \Delta_{i j}^{\alpha}+\sum_{\substack{j<i \leq k \\ \alpha}} \Delta_{j i}^{\alpha}\right) .
$$

$\mathbb{H}_{j}^{k}$ have perfect meaning on $S_{k}^{-}$and moreover, we have

$$
\left(\mathbb{H}_{j}^{k} F\right)\left(s^{+} s^{-}\right)=\left(\mathbb{H}_{j}^{k} F_{s^{+}}\right)\left(s^{-}\right),
$$

where $F_{s^{+}}\left(s^{-}\right)=F\left(s^{+} s^{-}\right)$is a function on $S_{k}^{-}$. In (D.38) $\mathbb{H}_{j}^{k}$ on the left-hand side is considered on $S$, while on the right hand side on $S_{k}^{-}$. Finally, $\mathbb{H}_{j}^{k}$ are the strongly diagonal Hua operators on $S_{k}^{-}$. By induction the left-hand side of (D.38) vanishes so

$$
\mathbb{H}_{j}^{k} K_{\psi}\left(s^{-}\right)=0 .
$$

Now weak regularity of $K_{\psi}$ implies that

$$
\Delta_{i k}^{\alpha} K_{\psi}=0, \quad \Delta_{k k} K_{\psi}=0 .
$$

But

$$
\Delta_{i k}^{\alpha} K_{\psi}\left(s^{-}\right)=\int_{S_{k}^{+}}\left(\Delta_{i k}^{\alpha} F\right)\left(s^{+} s^{-}\right) \psi\left(s^{+}\right) d s^{+}
$$

and so $\Delta_{i k}^{\alpha} F=0$. 


\section{REFERENCES}

[BBDhPt] A. Bonami, D. Buraczewski, E. Damek, A. Hulanicki, R. Penney, B. Trojan Hua system and pluriharmonicity for symmetric irreducible Siegel domains of type II, J. Funct. Anal. 188 (2002), 38-74.

[BBG] A. Bonami, J. Bruna, S. Grellier On Hardy, BMO and Lipschitz spaces of invariant harmonic functions in the unit ball, Proc. of the London Math. Soc. 71 (1998), 665-696.

[B] D. Buraczewski The Hua system on irreducible Hermitian symmetric spaces of nontube type. To appear in Ann. I. Fourier.

[BDH] D. Buraczewski, E. DAmek, A. Hulanicki Bounded pluriharmonic functions on symmetric irreducible Siegel domains, Math. Z. 240 (2002), 169-195.

[DHMP] E. DameK, A. Hulanicki, D. Müller, M. Peloso Pluriharmonic $H^{2}$ functions on symmetric irreducible Siegel domains, Geom. Funct. Anal. 10 (2000), 1090-1117.

[FK] J. Faraut, A. Korányi Analysis On Symmetric Cones, Oxford Math. Mongraphs, Oxford Sc.Publ. Calderon Press, 1994.

[Fo] G. Folland Harmonic analysis in phase space. Princeton University Press, Princeton, NJ, 1989.

[Gr1] C. R. Graham The Dirichlet problem for the Bergman Laplacian. I omm. Partial Differential Equations, 8 (1983), 433-476.

[Gr2] C. R. Graham The Dirichlet problem for the Bergman Laplacian. II omm. Partial Differential Equations, 8 (1983), 563-641.

[Ja1] Ph. Jaming Harmonic functions on the real hyperbolic ball I : Boundary values and atomic decomposition of Hardy spaces. Coll. Math., 80 (1999), 63-82.

[Ja2] Рн. Jaming Harmonic functions on classical rank one balls. Boll. Unione Mat. Ital. 4-B, 8 2001, 685-702.

[L] M. Lassalle Algebres de Jordan et ensemble de Wallach, Invent. Math., 89 (1987), 375-393.

[L1] G. LAVILLE Fonctions pluriharmoniques et solution foundamentale d'un opérateur du quatrième ordre, Bull. de Sc. Mathematiques 101 (1977), 305-317.

[JK] K. Johnson, A. Korányi The Hua operators on bounded symmetric domains of tube type, Ann. of Math. 111 (1980), 589-608.

[St] E.M. STEIn Harmonic analysis: real-variable methods, orthogonality, and oscillatory integrals.. Princeton Mathematical Series, 43. Monographs in Harmonic Analysis, III. Princeton University Press, Princeton, NJ, 1993.

[Sz] G. Szegö Orthogonal polynomials, Amer. Math. Soc., Colloq. publ., Providence, RI, 1967.

[T] S. Thangavelu Harmonic analysis on the Heisenberg group. Birkhäuser, Boston, MA 1998.

[Tr] B. TROJAN Hua-harmonic functions on homogeneous Siegel domains, preprint.

A. Bonami \& P. Jaming, Université d'Orléans, Faculté des Sciences, Département de Mathématiques, BP 6759, F 45067 Orléans Cedex 2, France

E-mail address: bonami@labomath.univ-orleans.fr, jaming@labomath.univ-orleans.fr

D. Buraczewski, E. Damek \& A. Hulanicki, 50-384 Wroclaw, Pl. Grunwaldzki 2/4, Poland

E-mail address: dbura@math.uni.wroc.pl, edamek@math.uni.wroc.pl, hulanick@math.uni.wroc.pl 